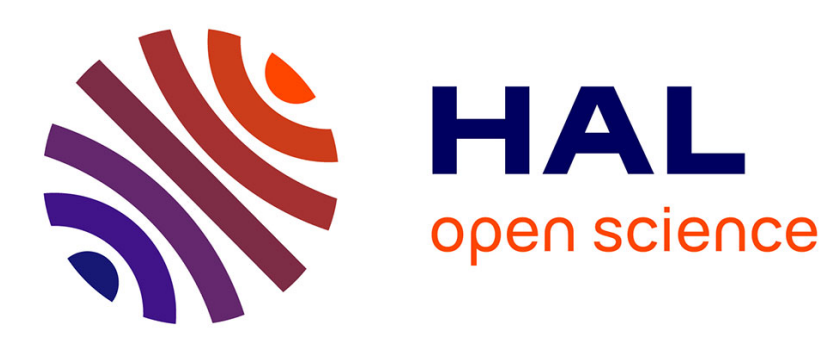

\title{
Comparison of multiphase models for computing shock-induced bubble collapse
}

Eric Goncalves da Silva, P. Parnaudeau

\section{To cite this version:}

Eric Goncalves da Silva, P. Parnaudeau. Comparison of multiphase models for computing shockinduced bubble collapse. International Journal of Numerical Methods for Heat and Fluid Flow, 2020, 30 (8), pp. 3845-3877. 10.1108/HFF-05-2019-0399 . hal-02407161

\section{HAL Id: hal-02407161 \\ https://hal.science/hal-02407161}

Submitted on 12 Nov 2021

HAL is a multi-disciplinary open access archive for the deposit and dissemination of scientific research documents, whether they are published or not. The documents may come from teaching and research institutions in France or abroad, or from public or private research centers.
L'archive ouverte pluridisciplinaire HAL, est destinée au dépôt et à la diffusion de documents scientifiques de niveau recherche, publiés ou non, émanant des établissements d'enseignement et de recherche français ou étrangers, des laboratoires publics ou privés. 


\section{Comparison of multiphase models for computing shock-induced bubble collapse}

Please cite as: E. Goncalves da Silva and P. Parnaudeau, "Comparison of multiphase models for computing shock-induced bubble collapse", International Journal of Numerical Methods for Heat \& Fluid Flow, Vol. 30, No. 8, pp. 3845-3877, 2020. Publisher: Emerald Publishing Limited. DOI: 10.1108/HFF-05-2019-0399

Deposit License: This author accepted manuscript is deposited under a Creative Commons Attribution Non-commercial 4.0 International (CC BY-NC) licence. This means that anyone may distribute, adapt, and build upon the work for non-commercial purposes, subject to full attribution. If you wish to use this manuscript for commercial purposes, please contact permissions@emerald.com.

Acknowledgments: This research was supported by FEDER project P-2017-BAFE-96 and ANR project ANR-18-CE46-009. Computations have been performed on the supercomputer facilities of the Mesocentre de Poitou-Charentes and the Mesocentre CRIANN. 


\section{Abstract}

Purpose The aim of this work is to quantify the relative importance of the multiphase model for the simulation of a gas bubble impacted by a normal shock wave in water. Both the free-field case and the collapse near a wall are investigated. Simulations are performed on both two-dimensional and three-dimensional configurations. The main phenomena involved in the bubble collapse are illustrated. A focus on the maximum pressure reached during the collapse is proposed.

Design/methodology/approach Simulations are performed using an inviscid compressible homogeneous solver based on different systems of equations. It consists in solving different mixture or phasic conservation laws and a transport-equation for the gas volume fraction. Three-dimensional configurations are considered for which an efficient massively parallel strategy was developped. The code is based on a Finite Volume discretization for which numerical fluxes are computed with a HLLC scheme.

Findings The comparison of three multiphase models is proposed. It is shown that a simple four-equation model is well-suited to simulate such strong shock-bubble interaction. The three-dimensional collapse near a wall is investigated. It is shown that the intensity of pressure peaks on the wall is drastically increased (more than 200\%) in comparison with the cylindrical case.

Originality Such a comparison of multiphase models in the case of a strong shock-induced bubble collapse is clearly original. Usually models are tested separately leading to a large dispersion of results. Moreover, simulations of a three-dimensional bubble collapse are scarce in the literature using such fine grids.

Keywords multiphase models; bubble collapse; 3-D simulation; shock waves 


\section{Introduction}

Cavitation erosion is a major problem for hydraulic and marine applications. This phenomenon occurs when vapor bubbles collapse in the vicinity of solid walls leading to negative consequences, such as vibrations, material damages and performance loss. On the other hand, the destructive effects of cavitation can be exploited in medical application such as shock wave lithotripsy (Jamaluddin et al., 2011). To clarify the physical mechanism, numerous experimental and numerical studies of the collapse of cavity in water have been proposed (Plesset and Chapman, 1971, Haas and Sturtevant, 1987, Bourne, 2002, Turangan et al., 2008, Johnsen and Colonius, 2009, Hawker and Ventikos, 2012, Ozlem et al., 2012). The bubble collapse close to the wall has been addressed as the fundamental mechanism producing damage. Its general behavior is characterized by the formation of a water jet that penetrates through the bubble and the generation of a blast wave during the induced collapse. Both the jet and the blast wave are possible damaging mechanisms. However, the high-speed dynamics, the small spatio-temporal scales as well as the complicated physics involved in these processes make any theoretical and experimental approach a challenge.

There are two typical frameworks for numerically simulating high-speed two-phase flows regarding how they treat the two-phase interface: the interface-tracking method or sharp interface methods and interface-capturing method or diffuse interface methods. Both approaches present advantages and drawbacks. With interface-tracking methods, the location of the interfaces, treated as sharp discontinuities, is explicitly represented during the time evolution (Ball et al., 2000, Hu et al., 2006, Nourgaliev et al., 2006, Terashima and Tryggvason, 2009, Lauer et al., 2012). On the other hand, diffuse interface methods are based on the solution of conservation laws (continuity, momentum, and energy equations), while relaxing the sharp character of material interfaces and thus allowing them to numerically diffuse over a small but finite region (Allaire et al., 2002, Shyue, 2006, Johnsen and Colonius, 2009, Coralic and Colonius, 2013, Apazidis, 2016). The computation of compressible multifluid flows lead to several difficulties due to non- 
physical oscillations generated at material interfaces when conservative schemes are used (Abgrall, 1996). The volume fraction variation across acoustic waves causes difficulties for the Riemann problem resolution particularly in the derivation of approximate Riemann solvers. This is due to the occurrence of the large discontinuities of thermodynamic variables and equations of state involved at material interfaces. In addition to that, the non-monotonic behavior of the sound speed in the mixture causes inaccuracies in wave's transmission across interfaces.

Assuming all fluid components are described by a single velocity and a single pressure function, the flow can be described by the compressible Euler equations and its thermodynamics properties given by an equation of state (EOS). In order to identify each fluid, a variable $\phi$ is used. Various choices of $\phi$ have been suggested in the literature, depending on the model assumptions : the heat capacities ratio $\gamma$ (Abgrall, 1996, Abgrall et al., 2003, Janan and Marjani, 2007), the mass fraction of gas (Shyue, 1998, Terashima et al., 2013) or the level-set function (Nourgaliev et al., 2006, Lauer et al., 2012). For these models, some single-fluid algorithms and quasi-conservative or non-conservative approaches were suggested to preserve the oscillation-free property. Recently, a non-oscillatory energysplitting conservative algorithm has been proposed to circumvent the appearance of oscillations around material interfaces (Lei and Li, 2018).

Recent developments in computing for compressible multiphase flows originated from the seven-equation two-phase flow model (Baer and Nunziato, 1986). The two-fluid approach is the most complete and each phase is governed by its own set of conservation laws. The additional volume fraction equation prevents the occurrence of spurious pressure oscillations (Utkin, 2019). For many problems of practical importance, a reduced five-equation model has been derived with the assumptions of velocity and pressure equilibrium between phases (Kapila et al., 2001, Murrone and Guillard, 2005, Daude et al., 2014) and applied with success in various flows. By assuming the thermal equilibrium between phases, a four-equation model can be expressed. It is composed of three conservation laws for mixture quantities completed by an equation for a non-conservative quantity describing the flow topology, usually the void ratio (Kunz et al., 2000, Goncalves and 
Charriere, 2014, Goncalves and Zeidan, 2017) or the mass fraction of one phase (Saurel et al., 2016). With the assumption of complete thermodynamic equilibrium between phases (local temperature, pressure, and free Gibbs enthalpy equality between phases), the three-equation models or Homogeneous Equilibrium Models (HEM) are derived (Xie et al., 2006, Goncalves and Patella, 2009, Bilanceri et al., 2010).

The present work focuses on the comparison of three one-fluid compressible models to simulate the collapse of a gas bubble impacted by a shock wave in water. This study is motivated by the large dispersion of results in the literature and the need to quantify the relative importance of the model. The considered approaches are: i) a five-equation model similar to the Kapila formulation (Kapila et al., 2001), ii) a four-equation model (Goncalves and Charriere, 2014, Goncalves and Zeidan, 2018) used for different two-phase applications involving cavitation, and iii) a multicomponent formulation (also called $\gamma$ model) based on the works of Johnsen (Beig and Johnsen, 2015). The same numerical methods are used for the spatial and time integration. We consider the case investigated experimentally by Bourne and Field (Bourne and Field, 1992) and numerically by Ball et al. (Ball et al., 2000). This case was computed by a large number of authors (Hu et al., 2006, Nourgaliev et al., 2006, Terashima and Tryggvason, 2009, Lauer et al., 2012, Hawker and Ventikos, 2012). Firstly, the free-field collapse is investigated with particular consideration on the maximum pressure reaching during the process. Secondly, the collapse near a rigid wall is computed and the evolution of the wall pressure is analysed. Both two- and three-dimensional simulations are performed. As commented by different authors (Hawker and Ventikos, 2012), the collapse process of a spherical bubble is faster and more intense in comparison with the $2 \mathrm{D}$ cylindrical case. In the present study, we show that the maximum pressure peak monitored at the wall is extremely higher for the spherical collapse, suggesting stresses and potential damage on the material.

The paper is organized as follows. In section 2, we first review the theoretical formulation and models. The description of numerical methods is presented in section 3 . 
Preliminary results are proposed in section 4 to attest the ability of the numerical tool to predict a shock-bubble interaction by comparison with experimental data. Then, results are proposed in the two following sections for a gas bubble impacted by a shock wave in water. The free-field case is studied in section 5 and the collapse near a rigid wall is investigated in section 6. Finally, conclusions and future works are discussed.

\section{Governing equations and models}

The numerical simulations are carried out using an in-house, two-phase code solving various one-fluid compressible inviscid systems. The phases are assumed to be sufficiently well mixed and the sizes of the dispersed particle are sufficiently small, thereby eliminating any significant relative motion. The phases are strongly coupled and move at the same velocity. In addition, the phases are assumed to be in mechanical equilibrium: they share the same pressure $P$. In case of assumption of thermal equilibrium, the phases share the same temperature $T$. We introduce $\alpha$ and $Y$ the void fraction and mass fraction of gas, respectively.

\subsection{The pure phases EOS}

In the present study, we used the convex stiffened gas EOS for the pure phases (see Metayer et al. (2004)):

$$
\begin{aligned}
P(\rho, e) & =(\gamma-1) \rho(e-q)-\gamma P_{\infty} \\
P(\rho, T) & =\rho(\gamma-1) C_{v} T-P_{\infty} \\
T(\rho, h) & =\frac{h-q}{C_{p}}
\end{aligned}
$$

where $e$ is the internal energy, $h$ the enthalpy, $\gamma=C_{p} / C_{v}$ is the heat capacity ratio, $C_{p}$ and $C_{v}$ are thermal capacities, $q$ the energy of the fluid at a given reference state and $P_{\infty}$ is a constant reference pressure. The speed of sound $c$ is given by:

$$
c^{2}=\gamma \frac{P+P_{\infty}}{\rho}=(\gamma-1)(h-q)
$$




\subsection{A five-equation model}

We consider the 2-temperature model proposed by Kapila et al. (Kapila et al., 2001, Murrone and Guillard, 2005) and used by various authors (Saurel et al., 2008, Daude et al., 2014). The model consists in mixture balance laws for momentum and energy, balance laws for mass of each pure phase and an additional equation for the void ratio. Viscosity, surface tension, and phase change are not expected to affect the bubble dynamics over the major part of the collapse and are therefore ignored. We present below the inviscid equations, expressed in variables $\left((1-\alpha) \rho_{l}, \alpha \rho_{v}, \rho \vec{V}, \rho E, \alpha\right)$ :

$$
\begin{aligned}
\frac{\partial\left((1-\alpha) \rho_{l}\right)}{\partial t}+\operatorname{div}\left((1-\alpha) \rho_{l} \vec{V}\right) & =0 \\
\frac{\partial\left(\alpha \rho_{v}\right)}{\partial t}+\operatorname{div}\left(\alpha \rho_{v} \vec{V}\right) & =0 \\
\frac{\partial(\rho \vec{V})}{\partial t}+\operatorname{div}\left(\rho \vec{V} \otimes \vec{V}+P I_{\mathrm{d}}\right) & =0 \\
\frac{\partial(\rho E)}{\partial t}+\operatorname{div}(\rho \vec{V} H) & =0 \\
\frac{\partial \alpha}{\partial t}+\vec{V} \cdot \operatorname{grad}(\alpha) & =\underbrace{\left.\left(\frac{\rho_{l} c_{l}^{2}-\rho_{v} c_{v}^{2}}{\rho_{l} c_{l}^{2}}\right)+\frac{\rho_{v} c_{v}^{2}}{\alpha}\right)}_{=K} \operatorname{div}(\vec{V})
\end{aligned}
$$

where $\vec{V}=(u, v)$ is the center of mass velocity vector, $E=e+V^{2} / 2$ denotes the total energy and $H=h+V^{2} / 2$ the total enthalpy. Subscripts 'l' and 'v' for liquid and vapor, respectively. The term $K$ involves the speed of sound of pure phases $c_{k}$ and it reflects the effects of changes in volume of each phase.

On the basis of the stiffened gas EOS for each pure phase, an expression for the pressure can be deduced from the mechanical equilibrium assumption (Saurel et al., 2008). This expression is available in all possible fluid states, as a function of the void fraction $\alpha$ and 
the vapour mass fraction $Y$ :

$$
\begin{aligned}
P(\rho, e, \alpha, Y) & =(\gamma(\alpha)-1) \rho(e-q(Y))-\gamma(\alpha) P_{\infty}(\alpha) \\
\frac{1}{\gamma(\alpha)-1} & =\frac{\alpha}{\gamma_{v}-1}+\frac{1-\alpha}{\gamma_{l}-1} \\
q(Y) & =Y q_{v}+(1-Y) q_{l} \\
P_{\infty}(\alpha) & =\frac{\gamma(\alpha)-1}{\gamma(\alpha)}\left[\alpha \frac{\gamma_{v}}{\gamma_{v}-1} P_{\infty}^{v}+(1-\alpha) \frac{\gamma_{l}}{\gamma_{l}-1} P_{\infty}^{l}\right]
\end{aligned}
$$

Temperature of both phases $T_{l}$ and $T_{v}$ follows the stiffened gas EOS.

Without heat and mass transfer, the mixture speed of sound obeys the Wallis or Wood formulation (Wallis, 1967). This speed is expressed as a weighted harmonic mean of speeds of sound of each phase:

$$
\frac{1}{\rho c_{\text {wallis }}^{2}}=\frac{\alpha}{\rho_{v} c_{v}^{2}}+\frac{1-\alpha}{\rho_{l} c_{l}^{2}}
$$

The five equations form a system having a hyperbolic nature. The eigenvalues of the 1-D system are:

$\lambda_{1}=u-c_{w a l l i s}, \lambda_{2,3,4}=u$ and $\lambda_{5}=u+c_{\text {wallis }}$.

\subsection{A four-equation model}

We modify the previous model assuming the thermal equilibrium between phases and that the liquid is at its saturation state. The model consists in three conservation laws for mixture quantities (mass, momentum and total energy) and the additional equation for the void fraction (Goncalves, 2013, Goncalves and Charriere, 2014):

$$
\begin{aligned}
\frac{\partial \rho}{\partial t}+\operatorname{div}(\rho \vec{V}) & =0 \\
\frac{\partial(\rho \vec{V})}{\partial t}+\operatorname{div}\left(\rho \vec{V} \otimes \vec{V}+P I_{\mathrm{d}}\right) & =0 \\
\frac{\partial(\rho E)}{\partial t}+\operatorname{div}(\rho \vec{V} H) & =0 \\
\frac{\partial \alpha}{\partial t}+\vec{V} \cdot \operatorname{grad}(\alpha) & =K \operatorname{div}(\vec{V})
\end{aligned}
$$


The mixture pressure follows equations (10)-(13). An expression for the mixture temperature can be deduced from the thermal equilibrium assumption:

$$
\begin{aligned}
T(\rho, h, Y) & =\frac{h_{l}-q_{l}}{C_{p_{l}}}=\frac{h_{v}-q_{v}}{C_{p_{v}}}=\frac{h-q(Y)}{C_{p}(Y)} \\
q(Y) & =Y q_{v}+(1-Y) q_{l} \\
C_{p}(Y) & =Y C_{p_{v}}+(1-Y) C_{p_{l}}
\end{aligned}
$$

The eigenvalues of the matrix of the system can be easily computed. The system is hyperbolic, eigenvalues for 1-D are:

$\lambda_{1}=u-c_{\text {wallis }}, \lambda_{2,3}=u$ and $\lambda_{4}=u+c_{\text {wallis }}$.

\subsection{A multicomponent model}

Different expressions have been proposed for the simulations of compressible multicomponent two-phase flows (Abgrall, 1996, Shyue, 1998, Abgrall and Karni, 2001, Johnsen and Colonius, 2009, Terashima et al., 2013). We consider here a variant of the formulation developed to prevent temperature errors (Beig and Johnsen, 2015). The Euler equations for multicomponent gases are written as

$$
\begin{aligned}
\frac{\partial \rho}{\partial t}+\operatorname{div}(\vec{V}) & =0 \\
\frac{\partial(\rho \vec{V})}{\partial t}+\operatorname{div}\left(\rho \vec{V} \otimes \vec{V}+P I_{\mathrm{d}}\right) & =0 \\
\frac{\partial(\rho E)}{\partial t}+\operatorname{div}(\rho \vec{V} H) & =0 \\
\frac{\partial\left(\alpha \rho_{v}\right)}{\partial t}+\operatorname{div}\left(\alpha \rho_{v} \vec{V}\right) & =0
\end{aligned}
$$

The pressure and the temperature are related to the other variables by the equation of state. For simplicity reasons, we rewrite the stiffened gas EOS as:

$$
\begin{aligned}
\rho(e-q) & =\frac{P}{\gamma-1}+\frac{\gamma P_{\infty}}{\gamma-1} \\
& =\rho C_{v} T+P_{\infty}
\end{aligned}
$$

For multiphase flows, a one-fluid formulation is followed, in which the same thermodynamic relationship holds in the entire domain. Material interfaces are denoted by changes 
in the material properties, which are advected by the flow.

Transport equations for specific functions of the material properties entering the equation of state must be solved. It has been shown for an isolated interface advection problem that, to maintain pressure and temperature equilibria in time and space, the transport equations for $1 /(\gamma-1), \gamma P_{\infty} /(\gamma-1)$ and $P_{\infty}$ must be solved in non-conservative form and that for $q$ and $C_{v}$ in conservative form (Beig and Johnsen, 2015). Pressure and temperature must be computed from these specific quantities in equations (26) and (27).

The complete system is therefore composed by 9 equations:

$$
\begin{aligned}
\frac{\partial \rho}{\partial t}+\operatorname{div}(\vec{V}) & =0 \\
\frac{\partial(\rho \vec{V})}{\partial t}+\operatorname{div}\left(\rho \vec{V} \otimes \vec{V}+P I_{\mathrm{d}}\right) & =0 \\
\frac{\partial(\rho E)}{\partial t}+\operatorname{div}(\rho \vec{V} H) & =0 \\
\frac{\partial\left(\alpha \rho_{v}\right)}{\partial t}+\operatorname{div}\left(\alpha \rho_{v} \vec{V}\right) & =0 \\
\frac{\partial[1 /(\gamma-1)]}{\partial t}+\vec{V} \cdot \operatorname{grad}(1 /(\gamma-1)) & =0 \\
\frac{\left.\partial P_{\infty} /(\gamma-1)\right]}{\partial t}+\vec{V} \cdot \operatorname{grad}\left(\gamma P_{\infty} /(\gamma-1)\right) & =0 \\
\frac{\partial P}{\partial t}+\vec{V} \cdot \operatorname{grad}\left(P_{\infty}\right) & =0 \\
\frac{\partial(\rho q)}{\partial t}+\operatorname{div}(\rho \vec{V} q) & =0 \\
\frac{\partial\left(\rho C_{v}\right)}{\partial t}+\operatorname{div}\left(\rho \vec{V} C_{v}\right) & =0
\end{aligned}
$$

The system is hyperbolic. In the 1-D case, the eigenvalues are:

$\lambda_{1}=u-c, \lambda_{2,3,4,5,6,7,8}=u$ and $\lambda_{9}=u+c$.

where the sound speed is defined by relation (4).

\section{$3 \quad$ Numerics}

The numerical simulations are carried out using an explicit solver for structured meshes. This solver is based on a cell-centered finite-volume discretisation (Goncalves and Zeidan, 
2018).

\subsection{Space discretisation}

In two-dimensional space, the different systems can be represented in a matrix form as:

$$
\frac{\partial U}{\partial t}+\operatorname{div}[G(U)]+B(U) \operatorname{div} \vec{V}=0
$$

- For the four-equation model, we have

$$
U=\left(\begin{array}{c}
\rho \\
\rho \vec{V} \\
\rho E \\
\alpha
\end{array}\right) ; \quad G(U)=\left(\begin{array}{c}
\rho \vec{V} \\
\rho \vec{V} \otimes \vec{V}+P I_{d} \\
\rho H \vec{V} \\
\alpha \vec{V}
\end{array}\right) \quad ; \quad B(U)=\left(\begin{array}{c}
0 \\
\overrightarrow{0} \\
0 \\
-(K+\alpha)
\end{array}\right)
$$

- For the five-equation model

$$
U=\left(\begin{array}{c}
(1-\alpha) \rho_{l} \\
\alpha \rho_{v} \\
\rho \vec{V} \\
\rho E \\
\alpha
\end{array}\right) \quad G(U)=\left(\begin{array}{c}
(1-\alpha) \rho_{l} \vec{V} \\
\alpha \rho_{v} \vec{V} \\
\rho \vec{V} \otimes \vec{V}+P I_{d} \\
\rho H \vec{V} \\
\alpha \vec{V}
\end{array}\right) \quad B(U)=\left(\begin{array}{c}
0 \\
0 \\
\overrightarrow{0} \\
0 \\
-(K+\alpha)
\end{array}\right)
$$

- For the multicomponent nine-equation model 
$U=\left(\begin{array}{c}\rho \\ \rho \vec{V} \\ \rho E \\ \alpha \\ 1 /(\gamma-1) \\ \gamma P_{\infty} /(\gamma-1) \\ P_{\infty} \\ \rho q \\ \rho C_{v}\end{array}\right) \quad G(U)=\left(\begin{array}{c}\rho \vec{V} \\ \rho \vec{V} \otimes \vec{V}+P I_{d} \\ \rho H \vec{V} \\ \alpha \vec{V} \\ \vec{V} /(\gamma-1) \\ \gamma P_{\infty} \vec{V} /(\gamma-1) \\ P_{\infty} \vec{V} \\ \rho q \vec{V} \\ \rho C_{v} \vec{V}\end{array}\right) \quad B(U)=\left(\begin{array}{c}0 \\ \overrightarrow{0} \\ 0 \\ -\alpha \\ -1 /(\gamma-1) \\ -\gamma P_{\infty} /(\gamma-1) \\ -P_{\infty} \\ 0 \\ 0\end{array}\right)$

We focus herein on finite volume schemes. Regular meshes are considered. Integrating the system on the cell $\mathcal{C}_{i}$ gives

$$
\Omega_{i} \frac{\partial U_{i}}{\partial t}+\sum_{\ell \in \partial \mathcal{C}_{i}} \int_{\ell} G(U) \cdot \vec{n}_{i, \ell} d \ell+\int_{\mathcal{C}_{i}} B(U) \operatorname{div} \vec{V} d S=0
$$

where $\partial \mathcal{C}_{i}$ is the boundary of the cell, $\Omega_{i}$ the surface of the cell and $\vec{n}_{i, \ell}$ is the outward normal (with respect to cell $\mathcal{C}_{i}$ ) of cell interface $\ell$.

The numerical flux through the cell interface is computed with a HLLC scheme (Toro et al., 1994, Batten et al., 1997). The method considers two averaged intermediate states $U_{L}^{*}$ et $U_{R}^{*}$ separated by the contact wave of speed $S_{M}$. The numerical flux $\Phi_{i, \ell}$ at cell interface $\ell$ can be expressed as:

$$
\Phi_{i, \ell}\left(U_{L}, U_{R}\right)=\left\{\begin{array}{lll}
G\left(U_{L}\right) \vec{n}_{i, \ell} & \text { if } \quad S_{L}>0 \\
G\left(U_{L}^{*}\right) \vec{n}_{i, \ell} & \text { if } \quad S_{L} \leq 0<S_{M} \\
G\left(U_{R}^{*}\right) \vec{n}_{i, \ell} & \text { if } \quad S_{M} \leq 0 \leq S_{R} \\
G\left(U_{R}\right) \vec{n}_{i, \ell} & \text { if } \quad S_{R}<0
\end{array}\right.
$$

where $S_{L}$ and $S_{R}$ are referred to the speeds of the smallest and largest waves at the cell interface. 
We introduce the normal velocity component $V_{n}=\vec{V} \cdot \vec{n}$. The left $(K=L)$ and right $(K=R)$ states of the variables $U_{K}^{*}$, and corresponding fluxes $G\left(U_{K}^{*}\right)$, are defined by the following relations:

- For the four-equation model

$$
\begin{gathered}
U_{K}^{*}=\left(\begin{array}{c}
\rho_{K}^{*} \\
(\rho u)_{K}^{*} \\
(\rho v)_{K}^{*} \\
(\rho E)_{K}^{*} \\
\alpha_{K}^{*}
\end{array}\right)=\frac{1}{S_{K}-S_{M}}\left(\begin{array}{c}
\rho_{K}\left(S_{K}-V_{n_{K}}\right) \\
(\rho u)_{K}\left(S_{K}-V_{n_{K}}\right)+\left(P^{*}-P_{K}\right) n_{i, \ell} \\
(\rho u)_{K}\left(S_{K}-V_{n_{K}}\right)+\left(P^{*}-P_{K}\right) n_{i, \ell} \\
(\rho E)_{K}\left(S_{K}-V_{n_{K}}\right)+P^{*} S_{M}-P_{K} V_{n_{K}} \\
\alpha_{K}\left(S_{K}-V_{n_{K}}\right) \\
\rho_{K}^{*} S_{M} \\
(\rho u)_{K}^{*} S_{M}+P^{*} n_{i, \ell} \\
(\rho v)_{K}^{*} S_{M}+P^{*} n_{i, \ell} \\
(\rho E)_{K}^{*} S_{M}+P^{*} S_{M} \\
\alpha_{K}^{*} S_{M}
\end{array}\right)
\end{gathered}
$$

- For the five-equation model

$$
U_{K}^{*}=\left(\begin{array}{c}
{\left[(1-\alpha) \rho_{l}\right]_{K}^{*}} \\
{\left[\alpha \rho_{v}\right]_{K}^{*}} \\
(\rho u)_{K}^{*} \\
(\rho v)_{K}^{*} \\
(\rho E)_{K}^{*} \\
\alpha_{K}^{*}
\end{array}\right)=\frac{1}{S_{K}-S_{M}}\left(\begin{array}{c}
(1-\alpha) \rho_{l K}\left(S_{K}-V_{n_{K}}\right) \\
\alpha \rho_{v K}\left(S_{K}-V_{n_{K}}\right) \\
(\rho u)_{K}\left(S_{K}-V_{n_{K}}\right)+\left(P^{*}-P_{K}\right) n_{i, \ell} \\
(\rho u)_{K}\left(S_{K}-V_{n_{K}}\right)+\left(P^{*}-P_{K}\right) n_{i, \ell} \\
(\rho E)_{K}\left(S_{K}-V_{n_{K}}\right)+P^{*} S_{M}-P_{K} V_{n_{K}} \\
\alpha_{K}\left(S_{K}-V_{n_{K}}\right)
\end{array}\right)
$$




$$
G\left(U_{K}^{*}\right) \vec{n}_{i, l}=\left(\begin{array}{c}
{\left[(1-\alpha) \rho_{l}\right]_{K}^{*} S_{M}} \\
{\left[\alpha \rho_{v}\right]_{K}^{*} S_{M}} \\
(\rho u)_{K}^{*} S_{M}+P^{*} n_{i, \ell} \\
(\rho v)_{K}^{*} S_{M}+P^{*} n_{i, \ell} \\
(\rho E)_{K}^{*} S_{M}+P^{*} S_{M} \\
\alpha_{K}^{*} S_{M}
\end{array}\right)
$$

- For the multicomponent model

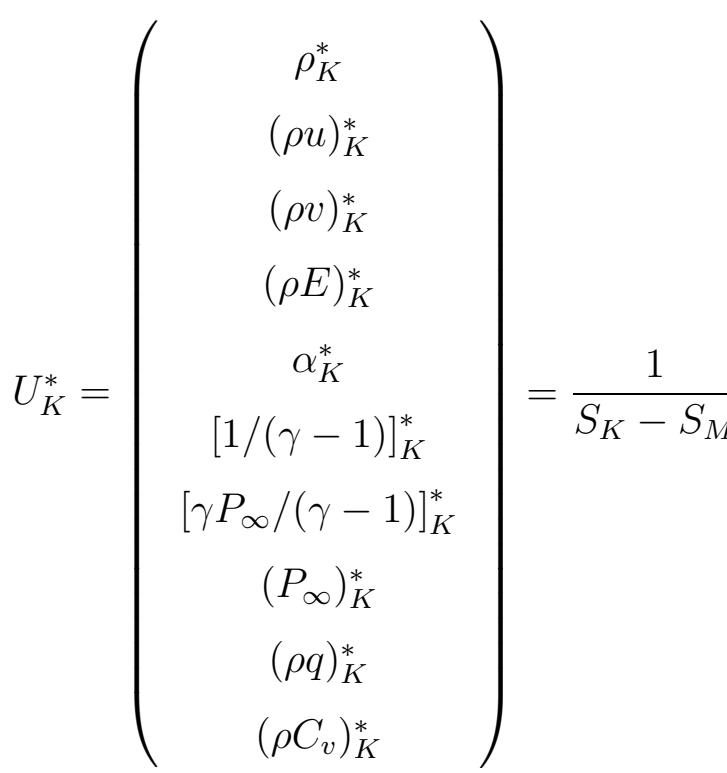

$$
\left(\begin{array}{c}
\rho_{K}\left(S_{K}-V_{n_{K}}\right) \\
(\rho u)_{K}\left(S_{K}-V_{n_{K}}\right)+\left(P^{*}-P_{K}\right) n_{i, \ell} \\
(\rho u)_{K}\left(S_{K}-V_{n_{K}}\right)+\left(P^{*}-P_{K}\right) n_{i, \ell} \\
(\rho E)_{K}\left(S_{K}-V_{n_{K}}\right)+P^{*} S_{M}-P_{K} V_{n_{K}} \\
\alpha_{K}\left(S_{K}-V_{n_{K}}\right) \\
{[1 /(\gamma-1)]_{K}\left(S_{K}-V_{n_{K}}\right)} \\
{\left[\gamma P_{\infty} /(\gamma-1)\right]_{K}\left(S_{K}-V_{n_{K}}\right)} \\
P_{\infty K}\left(S_{K}-V_{n_{K}}\right) \\
(\rho q)_{K}\left(S_{K}-V_{n_{K}}\right) \\
\left(\rho C_{v}\right)_{K}\left(S_{K}-V_{n_{K}}\right)
\end{array}\right)
$$

$$
G\left(U_{K}^{*}\right) \vec{n}_{i, l}=\left(\begin{array}{c}
\rho_{K}^{*} S_{M} \\
(\rho u)_{K}^{*} S_{M}+P^{*} n_{i, \ell} \\
(\rho v)_{K}^{*} S_{M}+P^{*} n_{i, \ell} \\
(\rho E)_{K}^{*} S_{M}+P^{*} S_{M} \\
\alpha_{K}^{*} S_{M} \\
{[1 /(\gamma-1)]_{K}^{*} S_{M}} \\
{\left[\gamma P_{\infty} /(\gamma-1)\right]_{K}^{*} S_{M}} \\
\left(P_{\infty}\right)_{K}^{*} S_{M} \\
(\rho q)_{K}^{*} S_{M} \\
\left(\rho C_{v}\right)_{K}^{*} S_{M}
\end{array}\right)
$$


where the pressure $P^{*}$ is given by:

$$
P^{*}=P_{L}+\rho_{L}\left(V_{n_{L}}-S_{L}\right)\left(V_{n_{L}}-S_{M}\right)=P_{R}+\rho_{R}\left(V_{n_{R}}-S_{R}\right)\left(V_{n_{R}}-S_{M}\right)
$$

And the contact-wave speed $S_{M}$ is defined by:

$$
S_{M}=\frac{P_{R}-P_{L}+\rho_{L} V_{n_{L}}\left(S_{L}-V_{n_{L}}\right)-\rho_{R} V_{n_{R}}\left(S_{R}-V_{n_{R}}\right)}{\rho_{L}\left(S_{L}-V_{n_{L}}\right)-\rho_{R}\left(S_{R}-V_{n_{R}}\right)}
$$

The HLLC solver requires the estimates of wave speeds $S_{L}$ and $S_{R}$ in the Riemann problem. A direct and simple wave speed estimation is used:

$$
S_{L}=\operatorname{Min}\left(V_{n_{L}}-c_{L}, V_{n_{R}}-c_{R}\right) \quad ; \quad S_{R}=\operatorname{Max}\left(V_{n_{L}}+c_{L}, V_{n_{R}}+c_{R}\right)
$$

For the non-conservative term, the integral term is approximated with the following relation:

$$
\int_{\mathcal{C}_{i}} B(U) \operatorname{div} \vec{V} d S=\tilde{B}_{i} \sum_{\ell \in \partial \mathcal{C}_{i}} \int_{\partial \mathcal{C}_{i}} \vec{V} \cdot \vec{n}_{i, \ell} d \ell
$$

where $\tilde{B}_{i}$ is some average of $B$ on cell $\mathcal{C}_{i}$. In this work, we have used $\tilde{B}_{i}=B\left(U_{i}\right)$.

The cell interface value $u_{i, \ell}$ is expressed as:

$$
u_{i, \ell}\left(U_{L}, U_{R}\right)=\left\{\begin{array}{clc}
\vec{V}_{L} \cdot \vec{n}_{i, \ell} & \text { if } \quad S_{L}>0 \\
\frac{S_{L}-V_{n}}{S_{L}-S_{M}} S_{M} & \text { if } \quad S_{L} \leq 0<S_{M} \\
\frac{S_{R}-V_{n}}{S_{R}-S_{M}} S_{M} & \text { if } \quad S_{M} \leq 0 \leq S_{R} \\
\vec{V}_{R} \cdot \vec{n}_{i, \ell} & \text { if } \quad S_{R}<0
\end{array}\right.
$$

The second-order accuracy in space is obtained using the MUSCL extrapolation. The minmod slope limiter has been chosen for all simulations. The temporal integration is performed using a three-step Runge-Kutta method.

\subsection{Inlet and outlet boundary conditions}

The numerical treatment of the boundary conditions is based on the use of the characteristic relations of the Euler equations. The number of variables to impose at boundaries is 
given by the number of positive characteristics directed into the domain of interest. The characteristic relations obtained for the five-equation system, in two-dimensional flows, are:

$$
\begin{aligned}
-c^{2}\left(\rho^{c}-\rho^{s}\right)+\left(P^{c}-P^{s}\right) & =0 \\
V_{t}^{c}-V_{t}^{s} & =0 \\
\rho\left(\alpha^{c}-\alpha^{s}\right)-K\left(\rho^{c}-\rho^{s}\right) & =0 \\
\left(Y^{c}-Y^{s}\right) & =0 \\
\left(P^{c}-P^{s}\right)+\rho c\left(V_{n}^{c}-V_{n}^{s}\right) & =0 \\
\left(P^{c}-P^{s}\right)-\rho c\left(V_{n}^{c}-V_{n}^{s}\right) & =0
\end{aligned}
$$

The variables with superscript $c$ denote the variables to be computed at the boundary. Variables with superscript $s$ denote the variables obtained by the current numerical scheme. $V_{t}$ and $V_{n}$ are the tangential and the normal component of the velocity, respectively.

At inflow, we impose the initial values of the void ratio, densities of pure phases, and the velocity. The pressure is evaluated with the last relation (48) and all variables can be evaluated at the boundary.

At the outflow, the static pressure is imposed. The variables are computed with all characteristic relations except the first one.

\section{Preliminary results}

A preliminary study is proposed to test the present numerical tool by comparison with experimental results. Due to the weak number of experimental studies on liquid shockbubble interaction, we make comparisons with a helium bubble immersed in air and impacted by a planar shock wave. Interaction of shocks in air with cylindrical and spherical cavities filled with helium or R22 gas were investigated in an early experimental study 
by Haas and Sturtevant (Haas and Sturtevant, 1987). More recently, an extensive experimental study of a shock impact on spherical helium bubbles was conducted by Layes et al. (Layes et al., 2009). We test the different models on one of theses cases. We consider a helium bubble, for which the initial diameter is $D_{0}=4 \mathrm{~cm}$, impacted by a normal shock wave moving at the Mach number $M_{s h}=1.175$. The volume fraction $\alpha$ in this case is the volume fraction of the lighter gas in a carrier gas. Due to the symmetry of the problem the calculations are performed in a half-domain. Layes et al. present an experimental investigation of the evolution of the bubble elongation $L$ to the original diameter $D_{0}$ as function of time. A schematic diagram of the definition of $L$ and a view of the computation domain of size $40 \times 4 \mathrm{~cm}$ are given in Figure 1. Parameters of the EOSs and post-shock conditions are:

$$
\begin{aligned}
\left(\begin{array}{c}
\gamma \\
P_{\infty} \\
\rho
\end{array}\right)_{\text {Air }}=\left(\begin{array}{c}
1.4 \\
0 \mathrm{~Pa} \\
1.163 \mathrm{~kg} / \mathrm{m}^{3}
\end{array}\right) ;\left(\begin{array}{c}
\gamma \\
P_{\infty} \\
\rho
\end{array}\right)_{\text {Helium }}=\left(\begin{array}{c}
1.648 \\
0 \mathrm{~Pa} \\
0.16 \mathrm{~kg} / \mathrm{m}^{3}
\end{array}\right) \\
\left(\begin{array}{c}
\mathrm{P} \\
\rho \\
\mathrm{u}
\end{array}\right)_{\text {post-shock }}=\left(\begin{array}{c}
1.44410^{5} \mathrm{~Pa} \\
1.51 \mathrm{~kg} / \mathrm{m}^{3} \\
93.65 \mathrm{~m} / \mathrm{s}
\end{array}\right)
\end{aligned}
$$

Simulations are performed using a uniform mesh composed by $2000 \times 200$ cells and a time step $\Delta t=510^{-9} \mathrm{~s}$. A three-dimensional simulation is also performed using the 4-equation model. Due to the relatively good symmetry of the bubble during its deformation observed in (Layes et al., 2009), only a quarter of the bubble is simulated with symmetry conditions. The mesh is extruded in the spanwise direction and is composed of $2000 \times 200 \times 200$ cells. A reference time $t_{0}=D_{0} / u_{\text {post-shock }}$ is introduced. The evolution of the bubble elongation $L / D_{0}$ as a function of the dimensionless time $t / t_{0}$ is illustrated in Figure 2 for all considered models and the experimental data. The interface of the bubble is evaluated using a criterion based on the variation of the void fraction between two neighbouring cells. The threshold value is set to $40 \%$ of the maximum value of the void fraction estimated at each time step, in agreement with a graphical estimation of 
the bubble interface. All simulations reproduce correctly the change of morphology of the bubble. The bubble is firstly flattened in the direction of the shock propagation and becomes kidney shaped due to the formation of a high speed air jet at the upstream interface. The jet impingement on the downstream interface induces the formation of counter-rotating vortical structures responsible for the bubble elongation.

Concerning the model comparison for the cylindrical case, we can see that the 4- and 5-equation models provide a similar solution. Discrepancies between numerical and experimental values are observed close to the minimum value of $L / D_{0}$ in the initial stage of bubble acceleration. At a later time, the bubble elongation is pretty well predicted by both these models. The solution obtained with the multicomponent model follows the same behaviour at the beginning of the interaction. Yet, after time $t / t_{0}>2.5$, the ratio $L / D_{0}$ is under-estimated and the elongation process is not well captured.

Taking the 2D and 3D results together, we observe that the flattening process and the beginning of the bubble elongation is very well predicted by the $3 \mathrm{D}$ simulation, certainly due to the more intense jet speed in the spherical case. Later, when the helium bubble is transformed into a vortex ring, the ratio $L / D_{0}$ is over-estimated and the bubble elongation is more pronounced in the spherical case in comparison with the cylindrical case.

The evolution of the density gradient modulus obtained for the cylindrical case with all models are plotted in Figures 3 and 4 at different dimensionless times $t / t_{0}$. The computed wave patterns are in good agreement with the shock observations presented in (Haas and Sturtevant, 1987). At time $t / t_{0}=0.09$, the incident shock has impacted the helium bubble. A reflected wave is seen on the left and a transmitted wave propagates inside the bubble. Further, because of the small critical angle, a precursor shock wave and Mach stem form outside the bubble, generating a shock-on-shock interaction. The transmitted shock wave reaches the downstream interface of the helium bubble around $t / t_{0}=0.19$. Later, a secondary transmitted wave propagates outside the bubble tangentially connected to the primary transmitted wave while a reflected wave runs inside the bubble. This internal reflected wave emerges from the upstream interface as a back-scattered wave. 
At time $t / t_{0}=0.26$, the first reflected wave impacts the wall and reflects. Later, the bubble becomes kidney shape due to the air jet. The distortion and motion of the helium volume are clearly illustrated. When the jet impinges on the downstream interface, it spreads out laterally forming a pair of vortical structures. As commented previously, the 4- and 5-equation models provide a similar solution. Yet, discrepancies are noticeable with the multicomponent solution. The penetration of the air jet is less pronounced at times $t / t_{0}=0.94$ and 1.17 . The acceleration of the gas is weaker, leading to a gap on the position of the bubble centroid.

\section{$5 \quad$ Shock-induced bubble collapse in free field}

\subsection{Presentation of the case}

We consider the interaction of a single cavity in water with a 1.9 GPa shock. This problem has been investigated experimentally by Bourne and Field (Bourne and Field, 1992). We compare our results with the previous simulations of Ball et al. (Ball et al., 2000), Nourgaliev et al. (Nourgaliev et al., 2006) and Hawker and Ventikos (Hawker and Ventikos, 2012).

A cylindrical air bubble, for which the initial diameter $D_{0}=6 \mathrm{~mm}$, is immersed in a water

pool, under the following initial conditions: $\vec{V}=(0,0) \mathrm{m} / \mathrm{s}, P=10^{5} \mathrm{~Pa}, \rho_{\text {air }}=1 \mathrm{~kg} / \mathrm{m}^{3}$, and $\rho_{\text {water }}=1000 \mathrm{~kg} / \mathrm{m}^{3}$. Due to the symmetry of the problem the calculations are performed in a half-domain. The center of the bubble is located at $(9,0) \mathrm{mm}$ in the computational domain of size $24 \times 12 \mathrm{~mm}$. The bubble is collapsed by a normal shock wave moving at $M_{s h}=1.72$, initially located at abscissa $x_{s h}=4 \mathrm{~mm}$. Parameters of the EOSs and post-shock conditions are:

$$
\left(\begin{array}{c}
\gamma \\
P_{\infty} \\
\rho
\end{array}\right)_{\text {Liquid }}=\left(\begin{array}{c}
4.4 \\
6 \times 10^{8} \mathrm{~Pa} \\
1000 \mathrm{~kg} / \mathrm{m}^{3}
\end{array}\right) ;\left(\begin{array}{c}
\gamma \\
P_{\infty} \\
\rho
\end{array}\right)_{\mathrm{Gas}}=\left(\begin{array}{c}
1.4 \\
0 \mathrm{~Pa} \\
1 \mathrm{~kg} / \mathrm{m}^{3}
\end{array}\right)
$$




$$
\left(\begin{array}{l}
\mathrm{P} \\
\rho \\
\mathrm{u}
\end{array}\right)_{\text {post-shock }}=\left(\begin{array}{c}
1.910^{9} \mathrm{~Pa} \\
1323.65 \mathrm{~kg} / \mathrm{m}^{3} \\
681.58 \mathrm{~m} / \mathrm{s}
\end{array}\right)
$$

The boundary conditions are the following: the top and bottom boundaries are assumed to be a wall and a symmetry axis, respectively. The left and right sides are assumed to be non-reflecting. Simulations are performed using a uniform mesh composed by $1600 \times 800$ cells and a time step $\Delta t=10^{-9} \mathrm{~s}$. The influence of both time and space steps has been previously studied in (Goncalves et al., 2018) to ensure that grid independence was achieved.

Firstly, a description of the main phenomena involved in this shock-bubble interaction is proposed. Results are obtained using the five-equation model. The time evolution of the density gradient modulus (Schlieren-type representation) and the pressure field are plotted in Figure 5 from time $t=2.4 \mu \mathrm{s}$ to $t=4.8 \mu \mathrm{s}$. After the water shock wave has collided with the bubble, a strong rarefaction wave is reflected backwards from the interface, and a weak shock wave is transmitted inside the bubble (time $t=2.4 \mu \mathrm{s}$ ). Due to the pressure difference between both sides, the bubble is asymmetrically contracted (time $t=3 \mu \mathrm{s}$ ), which induces a jet of water along the axis of flow symmetry. When this water jet strikes the right interface of the bubble (at time $t=3.6 \mu \mathrm{s}$ ), an intense blast wave (referred to as water-hammer shock (Hawker and Ventikos, 2012)) is generated in the surrounding water leading to a high-pressure zone. The blast front, which expands continuously, is highly asymmetric due to the high-speed water jet (see at time $t=4$ $\mu \mathrm{s})$. The rightward blast wave increases as a cylindrical wave (time $t=4.4 \mu \mathrm{s}$ ). The interaction of the leftward wave with the bubble fragments leads to high pressure levels (around time $t=4.8 \mu \mathrm{s}$ ), which is the most intense reached during the collapse. 


\subsection{Comparison of models}

We compare the behaviour of the three considered models. The maximum pressure $P_{\max }$ reached during the collapse is plotted in Figure 6. The 4- and 5-equation models provide similar results. One can observe the first peak at time $t=3.8 \mu$ s after the water jet impacts the bubble front and the second peak at time $t=4.7 \mu$ s when the leftward blast wave collides the bubble fragments. Small discrepancies are noticeable on the peak values, which are slightly higher using the five-equation model. For the multicomponent model, although the global evolution is similar, some clear differences are illustrated. We observe a time shift for the pressure increases corresponding to the blast wave formation and the bubble pieces collapse. Moreover, the intensity of the maximum pressure peak is reduced in comparison with the other models. Values of these different times and peaks are given in Table 1. The second peak predicted with the multicomponent model is almost $20 \%$ smaller in comparison with the five-equation solution.

\begin{tabular}{cccc}
\hline & 4-equation & 5-equation & multicomponent \\
\hline blast wave formation time $(\mu \mathrm{s})$ & 3.54 & 3.54 & 3.59 \\
first peak time $(\mu \mathrm{s})$ & 3.8 & 3.8 & 4.2 \\
first peak intensity $(\mathrm{bar})$ & 43,000 & 48,000 & 43,000 \\
second peak time $(\mu \mathrm{s})$ & 4.7 & 4.7 & 4.78 \\
second peak intensity $(\mathrm{bar})$ & 77,200 & 80,000 & 66,700 \\
maximum jet velocity $(\mathrm{m} / \mathrm{s})$ & 3700 & 3500 & 3300 \\
temperature peak $(\mathrm{K})$ & 10,000 & - & 5,700 \\
\hline
\end{tabular}

Table 1: Comparison of results obtained with the three models.

The pressure evolution on the symmetry axis is plotted at different times in Figure 7 for results obtained with the four-equation model (left) and multicomponent model (right). As the results provided by the five-equation model are very close to the four-equation solution, they are not presented here. For the four-equation model, we can observe the first peak at time $t=3.8 \mu$ s after the water jet impacts the bubble front leading to the 
blast wave generation, the decrease of this peak at time $t=4.2 \mu$ s and the second peak (around 68,000 bar) at time $t=4.8 \mu$ s when the leftward blast wave collides with the bubble fragments. We remark that the maximum value of the pressure peak (around 77,200 bar, see Table 1) is not located on the symmetry axis but in the bubble pieces (see Fig. 5). For the multicomponent model, the pressure rise takes more time up to $t=4.2 \mu \mathrm{s}$. The intensity of the second peak on the axis (around 66,000 bar) is close to the maximum value $(66,700 \mathrm{bar})$. Moreover, this peak is located downstream $(x=0.0111$ $\mathrm{m})$ in comparison with the four-equation solution $(x=0.0104 \mathrm{~m})$.

The evolution of the axial velocity on the symmetry axis is drawn in Figure 8 for results obtained with the four-equation model (left) and multicomponent model (right). The acceleration of the flow leading to the high-speed jet is clearly illustrated for both models. The axial velocity reached its maximum value around time $t=3 \mu \mathrm{s}$, decreases and remains at a high level around $2500 \mathrm{~m} / \mathrm{s}$. The intensity of the jet is higher using the four-equation model $(3700 \mathrm{~m} / \mathrm{s})$ in comparison with the multicomponent model (3300 $\mathrm{m} / \mathrm{s}$ ). Our results are in agreement with the experimental work of Bourne and Field, who reported a jet velocity at impact around $3300 \pm 300 \mathrm{~m} / \mathrm{s}$.

The temperature evolution along the symmetry axis is plotted in Figure 9 for results obtained with the 4-equation model (left) and multicomponent model (right). We clearly observe two intense peaks at times 2 and $3 \mu \mathrm{s}$, respectively. They correspond to the temperature augmentation across the transmitted shock wave inside the bubble. These peaks are largely higher with the four-equation model: $6000 \mathrm{~K}$ for the first peak to compare with $1600 \mathrm{~K}$ for the multicomponent model. The more intense peak is observed just before the high-speed jet impinges upon the downstream side of the bubble. It reaches 10,000 K for the four-equation model and $5700 \mathrm{~K}$ for the multicomponent model. Different authors have discussed about the existence of extreme temperatures reached during the bubble collapse with the formation of a hot plasma linked to the sonoluminescence phenomenon (Flannigan and Suslick, 2005, Mahdi et al., 2010, Merouani et al., 2014). The temperature 
field obtained with the 5-equation model is not presented. Indeed, due to the absence of heat transfer model between phases, the gas temperature reaches huge values.

\begin{tabular}{llll}
\hline & Ball (2000) & Nourgaliev (2006) & Hawker \& Ventikos \\
\hline peak pressure on jet impact (bar) & 47,000 & 101,000 & 58,900 \\
jet velocity on jet impact (m/s) & 2600 & 2850 & 2810 \\
temperature peak $(\mathrm{K})$ & 12,000 & 25,000 & 14,000 \\
\hline
\end{tabular}

Table 2: Comparison with previous 2D simulations of the same configuration.

In comparison with previous numerical simulations presented in Table 2, we see that our results are globally coherent.

Finally, the computation cost is given in Table 3 for a simulation time of $6 \mu$ s (singleprocessor calculation). Due to the stiffness of the case, the time step has been reduced to $5 \cdot 10^{-10} \mathrm{~s}$ for the five-equation simulation, leading to a factor 3 for the CPU cost in comparison with the four-equation model. The multicomponent model induces a supplementary cost by a factor 1.6.

As regard to this test case, the four-equation model is clearly more attractive than the five-equation formulation: we obtain similar results for a weaker cost. Is is not easy to discriminate the four-equation and multicomponent models due to the absence of reference data.

\begin{tabular}{ccc}
\hline models & $\Delta t(\mathrm{~s})$ & $\mathrm{CPU}$ cost ratio \\
\hline 4-equation & $10^{-9}$ & 1 \\
5-equation & $5.10^{-10}$ & 2.95 \\
multicomponent & $10^{-9}$ & 1.61 \\
\hline
\end{tabular}

Table 3: Comparison of CPU costs for a 2D simulation time of $6 \mu \mathrm{s}$. 


\subsection{Three-dimensional simulation}

We consider now a three-dimensional simulation using the four-equation model. The domain is extruded in the spanwise direction. The center of the bubble is located at $(9,0,0) \mathrm{mm}$ in the computational domain of size $24 \times 12 \times 12 \mathrm{~mm}$, as illustrated in Figure 10. The mesh is composed of $1600 \times 800 \times 800$ cells. Due to the symmetry assumption, we compute only a quarter of the spherical bubble. The time step is set to $5 \cdot 10^{-10} \mathrm{~s}$. The simulations are performed at a resolution of 200 points per radius (ppr), which amounts to a total number of about 1 billion cells.

The evolution of the maximum pressure during the cavity collapse is plotted in Figure 11 for both 2D and 3D simulations. Main phenomena are similar to those described previously in the $2 \mathrm{D}$ case. One can observe the first peak after the water jet impacts the bubble front and the second peak (more intense) when the leftward blast wave collides the bubble fragments. The bubbles turn into toroids and the flow creates a ring vortex. As observed previously (Hawker and Ventikos, 2012), the collapse process of a spherical bubble is faster and more intense, resulting in higher pressure peaks. These authors estimated the water-hammer shock pressure to be $40 \%$ higher for a spherical bubble. Table 4 compares various quantitative measures between $2 \mathrm{D}$ and $3 \mathrm{D}$ simulations. The more intense peak reaches 111,000 bar for the 3D case (50\% more than the $2 \mathrm{D}$ collapse). These effects are due to the higher focusing that the 3D situation entails, which lead to a faster water jet.

\begin{tabular}{ccc}
\hline & 2-D case & 3-D case \\
\hline first peak time $(\mu \mathrm{s})$ & 3.8 & 3.4 \\
first peak intensity (bar) & 43,000 & 68,000 \\
second peak time $(\mu \mathrm{s})$ & 4.7 & 4.07 \\
second peak intensity (bar) & 77,200 & 111,100 \\
\hline
\end{tabular}

Table 4: Comparison of results from 2D and 3D simulations. 


\section{Bubble collapse near a wall}

This test is an extension of the last one considering a wall placed behind the bubble at an initial distance from the center $L$. The ratio $L / R$ is a major parameter that governs the bubble collapse dynamics. As suggested in (Johnsen and Colonius, 2009), the bubble initially located at a distance lower than $L / R=2$ presents high potential to cause damage. We consider the case for which the distance $L=5 \mathrm{~mm}(L / R=1.66)$. Numerical parameters are similar to the previous case. The size of the computational domain is $14 \times 12 \mathrm{~mm}$. Simulations are performed using a uniform mesh composed by $934 \times 800$ cells and a time step $\Delta t=10^{-9} \mathrm{~s}$.

\subsection{Four-equation simulation}

A qualitative description of the physical phenomena is proposed from results obtained using the four-equation model. At time $t=3.6 \mu \mathrm{s}$, as previously discussed, the water jet collides the bubble interface leading to the formation of an intense blast wave. At this same time, the incident shock impacts the wall and reflects, which generates the first pressure peaks on the solid (around 50,000 bar). The time evolution of the density gradient modulus and the pressure field are plotted in Figures 12 from time $t=4.2 \mu$ s to $t=6$ $\mu \mathrm{s}$. The rightward front of the blast wave impacts the wall at time $t=4.2 \mu \mathrm{s}$ and reflects as a reinforced shock wave generating a high-pressure area (time $t=4.4 \mu$ s and later). The pressure on the wall reaches more than 80,000 bar. The cylindrical reflected wave impacts the contact line between the fluid of the jet and that of the leeward bubble. A quasi normal front propagates between the bubble pieces, while the cylindrical part continues its progression (time $t=4.6 \mu \mathrm{s}$ ). The leftward front of the blast wave re-collapses the bubble fragments generating a high-pressure zone in the fluid and interacts with the other front of the blast wave (time $t=4.8 \mu \mathrm{s}$ and later). This re-collapse process of the remaining bubble generates another strong shock waves in the liquid, called sheet-jetting shocks (Hawker and Ventikos, 2012). These strong shocks propagate in all directions. The impact and reflection on the wall result in the most intense wall pressure peak at time 
$t=5 \mu$ s (around 110,000 bar, see Figure 13). The wave network is very complex with numerous interactions. At time $t=5.4 \mu \mathrm{s}$, a high-pressure zone is observed behind the leftward front on the symmetry axis. Finally, at time $t=6 \mu \mathrm{s}$, the contact line is close to impact the wall. The wall pressure is still high with values reaching 75,000 bar.

The evolution of the wall pressure on the symmetry axis at different times is shown in Figure 13 (on the left). At time $t=3.6 \mu \mathrm{s}$, the incident shock impacts the wall, which generates the first pressure peak. At time $t=4.2 \mu \mathrm{s}$, the blast wave impacts the wall and its reflection causes an intense pressure peak on the axis. At time $t=4.4 \mu \mathrm{s}$, the superposition of the reflected blast wave and the incident shock generates a highpressure area with a peak located around abscissa $x \simeq 0.0015 \mathrm{~m}$ (it can be observed in Figure 12b). Later, the maximum wall pressure value is again located on the symmetry axis. As previously discussed, the most intense peak is reached around time $t=5 \mu$ s due to the impact of sheet-jetting shocks on the wall. After this time, the pressure peak on this axis decreases but the high pressure area extends and covers all the wall surface at $t=7 \mu \mathrm{s}$. The maximum wall pressure reached during the collapse is plotted in Figure 13 (on the right). Numerous peaks can be observed, corresponding to the impact of various waves as previously described: the incident shock (time $t=3.6 \mu \mathrm{s}$ ), the blast wave (time $t=4.4 \mu \mathrm{s}$ ) and sheet-jetting shocks (time $t=5 \mu \mathrm{s}$ and also time $t=5.4 \mu \mathrm{s}$ ). We remark also that the maximum value, close to 107,000 bar, is not located on the axis but near the axis.

\subsection{Multicomponent simulation}

We compare now results obtained with the multicomponent model. The time evolution of the density gradient modulus and the pressure field are plotted in Figures 14 at different times. The main phenomena described previously are observed. As commented for the free-field case, a small lag is noticeable for the time of generation of the blast wave and therefore its impact on the wall. The shape of the collapsing bubble is not the same, and 
especially the contact line between the fluid of the jet and that of the leeward bubble, which is larger using the multicomponent model.

The evolution of both the wall pressure on the axis and the maximum wall pressure are shown in Figure 15. On the symmetry axis, we can see that the pressure peaks reach similar values between models. For both simulations, the maximum intensity is close to 100,000 bar at time $t=5 \mu \mathrm{s}$. The maximum wall pressure marks some difference. The pressure peak due to the blast wave reflection (around time $t=4.4 \mu \mathrm{s}$ ) is less pronounced. As previously commented, the maximum value (around 110,000 bar) is not located on the axis but close the axis. Moreover, the maximum value is no more obtained at time $t=5$ $\mu \mathrm{s}$ but at times $t=5.6 \mu \mathrm{s}$ and $t=6 \mu \mathrm{s}$ due to the sheet-jetting shocks (see Figure 16). For the free-field case, we remarked that the intensity of the pressure peak in the fluid was lower in comparison with the four-equation result (see Table 1: 66,700 bar in comparison with 77,200 bar). Yet, as regard to the wall pressure peaks, both models provide similar intensities.

An enlargement of the density gradient modulus and pressure field is proposed in Figure 16 in order to better observe the sheet-jetting shocks. At time $t=5.4 \mu \mathrm{s}$, these shock waves are well illustrated and propagate in both direction. The leftward wave impacts the wall and reflects at time $t=5.6 \mu$ s generating a high-pressure area. Another wave reflects on the wall at time $t=6 \mu$ generating the second high pressure peak highlighted in Figure 15.

\subsection{Three-dimensional simulation}

The computational domain is extruded in the spanwise direction, that is $14 \times 12 \times 12$ mm. The mesh contains $934 \times 800 \times 800$ cells. Due to the stiffness of the case, the time step is decreased to $10^{-10} \mathrm{~s}$. Only a simulation performed with the four-equation model is presented. 
The pressure field and isosurfaces of the density are plotted in Figure 17 at different times. At time $t=2.15 \mu \mathrm{s}$, the incident shock impacted the bubble and the water jet penetration is well illustrated. At time $t=3.25 \mu \mathrm{s}$, the blast wave is propagating after the water jet collided the bubble interface. The incident shock wave impacts the wall at time $t=3.65 \mu \mathrm{s}$ and the leftward blast wave propagates toward the vortex ring. The rightward blast front impacts the wall at time $t=3.9 \mu$ s leading to a high-pressure area on the wall. At time $t=4.15 \mu \mathrm{s}$, the low-pressure area associated to the vortex ring is clearly illustrated. The leftward blast front continues its propagation. At time $t=4.25$ $\mu \mathrm{s}$, a pressure peak is monitored at the wall due to the sheet-jetting shock waves (not visible on this figure).

Another view of the pressure field is given in Figure 18 to better observe the wall pressure evolution. A time $t=3.9 \mu \mathrm{s}$, the blast wave impacts the wall generating a highpressure area around the bubble axis until the incident shock wave impact has generated a pressure load on the wall. The reflection of the blast wave induces an intense pressure peak on the wall illustrated at time $t=4.15 \mu \mathrm{s}$. Another wall pressure peak around the bubble axis is observed at time $t=4.5 \mu$ s due to the impact of a sheet-jetting wave. At times $t=4.6$ and $t=4.75 \mu \mathrm{s}$, a high-pressure area is highlighted in the fluid inside the vortex ring. It is due to the reflected blast wave which recollapses the bubble fragments. The most intense wall pressure peak is observed between time $t=4.75$ and $t=4.85 \mu \mathrm{s}$ due to the impact and reflection of a sheet-jetting shock wave (see figure 19).

The evolution of the maximum wall pressure reached during the bubble collapse is plotted in Figure 19. The intense peak due to the blast wave impact (around 170,000 bar) is observed around time $t=3.9 \mu \mathrm{s}$. Later, two other peaks are illustrated due the impact of sheet-jetting waves (close to 150,000 bar and 125,000 bar, respectively). The most intense peak at time $t=4.78 \mu$ s is extremely violent (higher than 300,000 bar) due to another sheet-jetting shock wave. In comparison with the 2D solution, the intensity is 
multiplied by a factor 3 .

Finally, the wall pressure evolution on the symmetry axis is shown in Figure 20. One can observe the impact and reflection of different waves on the wall: the incident shock wave $(t=3.63 \mu \mathrm{s})$, the blast wave $(t=3.76-4.02 \mu \mathrm{s})$, a sheet-jetting wave $(t=4.26 \mu \mathrm{s})$ and the most intense peak at time $t=4.78 \mu \mathrm{s}$. Similarly to the $2 \mathrm{D}$ case, the largest values of the pressure are not located on the symmetry axis but around the longitudinal axis.

Now, we compare our simulations with an existing model of prediction of the maximum pressure during the collapse. Recently, a predictive framework for the peak pressure from nonspherical bubble collapses has been developped by Supponen et al. (Supponen et al., 2017) using simultaneous time-resolved shadowgraphy and hydrophone pressure measurements. Their model is based on the assumption that the shock wave is generated by a jet impact hammer pressure. The formulation involves the parameter $\zeta$, which represents the dimensionless equivalent of the Kelvin impulse and the distance $d$ between the bubble center and the hydrophone sensor:

$$
P_{\text {max }}=1.6\left(\rho c^{2} \Delta P\right)^{1 / 2}\left(\frac{R_{0}}{d}\right)^{1.2} \zeta^{-0.17} \quad ; \quad \zeta=0.195\left(\frac{L}{R_{0}}\right)^{-2}
$$

The application of the model for different values of the distance $d$ is given in Table 5 . A large discrepancy appears between the numerical results and the model prediction. This model has been built and compared with data obtained for weak driving pressures $\Delta P$. In the present study, the intensity of the incident shock wave is very strong $(\Delta P=1.9$ GPa), that could explain this gap.

\begin{tabular}{cccc}
\hline & $R_{0} / d=1 / 2$ & $R_{0} / d=1$ & $R_{0} / d=2$ \\
\hline$P_{\max }$ & 24,500 & 56,300 & 129,000 \\
\hline
\end{tabular}

Table 5: Prediction of the maximum pressure (in bar) given by the model of Supponen et al. when the ratio $R_{0} / d$ varies. 


\section{Conclusion}

In the present study, a comparison of two-phase models is proposed for the simulation of a shock-induced bubble collapse. Three different formulations are considered: a fiveequation model, a four-equation model and a $\gamma$-model. First, the free-field collapse is investigated. It is shown that the main phenomena are similarly computed by models. Yet, some clear differences are highlighted as regard to the intensity of the pressure peaks and their time of appearance. The $\gamma$-model provides a lower pressure intensity (20\% smaller for the most intense peak) with a time shift in comparison with the other models. The CPU cost comparison tends to eliminate the five-equation model in comparison with the four-equation one: it provides similar results with a cost multiplied by a factor 3 . A three-dimensional simulation is performed using the four-equation system. The collapse process of a spherical bubble is faster and more intense, resulting in a $50 \%$ more intense pressure peak in comparison with the cylindrical case.

Secondly, the bubble collapse near a wall is studied. The ratio $L / R$ between the bubble distance to the wall and the initial radius of the bubble is a key parameter. In this paper, the ratio is set to 1.66 leading to high potential damages. It is shown that the main phenomena are similarly computed by both the four-equation and $\gamma$-models. The pressure loading on the material surface during the bubble collapse is due to the impact of the blast wave and the sheet-jetting shock waves, which are generated by the collapse of the remaining bubble ring. The intensity of the most intense pressure peak has the same magnitude (around 110,000 bar) using both models.

Finally, a three-dimensional simulation is performed using the four-equation model. It is highlighted that the impact of the sheet-jetting shock wave leads to a huge pressure peak on the wall for which the intensity is higher than 300,000 bar (200\% more intense in comparison with the cylindrical case). This pressure loading can cause high stresses inside the material making the structure more vulnerable of having micro-ruptures. The pertinence of $2 \mathrm{D}$ simulations to study wall damages caused by a bubble collapse is therefore questionable for such cases. 
Further works are in progress to study the influence of the parameter $L / R$ on the collapse and to perform coupled fluid-structure simulations.

\section{Acknowledgments}

This research was supported by FEDER project P-2017-BAFE-96 and ANR project ANR-

18-CE46-009. Computations have been performed on the supercomputer facilities of the Mesocentre de Poitou-Charentes and the Mesocentre CRIANN. 


\section{References}

Abgrall, R. (1996), 'How to prevent pressure oscillations in multicomponent flow calculations : a quasi conservative approach', Journal of Computational Physics 125(1), 150160.

Abgrall, R. and Karni, S. (2001), 'Computations of compressible multifluid', Journal of Computational Physics 169(2), 594-623.

Abgrall, R., Nkonga, B. and Saurel, R. (2003), 'Efficient numerical approximation of compressible multi-material flow for unstructured meshes', Computers \& Fluids 32(4), 571605.

Allaire, G., Clerc, S. and Kokh, S. (2002), 'A five-equation model for the simulation of interfaces between compressible fluids', Journal of Computational Physics 181(2), 577616.

Apazidis, N. (2016), 'Numerical investigation of shock induced bubble collapse in water', Physics of Fluids 28, 046101.

Baer, M. and Nunziato, J. (1986), 'A two-phase mixture theory for the deflagration-todetonation transition (DDT) in reactive granular materials', Int. Journal of Multiphase Flow 12, 861-889.

Ball, G., Howell, B., Leighton, T. and Schofield, M. (2000), 'Shock-induced collapse of a cylindrical air cavity in water: a Free-Lagrange simulation', Shock Waves 10, 265-276.

Batten, P., Clarke, N., Lambert, C. and Causon, D. (1997), 'On the choice of wave speeds for the HLLC Riemann solver', SIAM J. Sci. Comput. 18(6), 1553-1570.

Beig, S. and Johnsen, E. (2015), 'Maintaining interface equilibrium conditions in compressible multiphase flows using interface capturing', Journal of Computational Physics $302,548-566$. 
Bilanceri, M., Beux, F. and Salvetti, M. (2010), 'An implicit low-diffusive HLL scheme with complete time linearization: application to cavitating barotropic flows', Computers E Fluids 39, 1990-2006.

Bourne, N. (2002), 'On the collapse of cavities', Shock Waves 11, 447-455.

Bourne, N. and Field, J. (1992), 'Shock-induced collapse of single cavities in liquids', Journal of Fluid Mechanics 244, 225-240.

Coralic, V. and Colonius, T. (2013), 'Shock-induced collapse of a bubble inside a deformable vessel', European Journal of Mechanics B/Fluids 40, 64-74.

Daude, F., Galon, P., Gao, Z. and Blaud, E. (2014), 'Numerical experiments using a HLLC-type scheme with ALE formulation for compressible two-phase flows fiveequation models with phase transition', Computer \&f Fluids 94, 112-138.

Flannigan, D. and Suslick, K. (2005), 'Plasma formation and temperature measurement during single-bubble cavitation', Nature (434), 52-55.

Goncalves, E. (2013), 'Numerical study of expansion tube problems: Toward the simulation of cavitation', Computers \& Fluids 72, 1-19.

Goncalves, E. and Charriere, B. (2014), 'Modelling for isothermal cavitation with a fourequation model', International Journal of Multiphase Flow 59, 54-72.

Goncalves, E. and Patella, R. F. (2009), 'Numerical simulation of cavitating flows with homogeneous models', Computers \& Fluids 38(9), 1682-1696.

Goncalves, E. and Zeidan, D. (2017), 'Numerical study of turbulent cavitating flows in thermal regime', International Journal of Numerical Method for Heat and Fluid Flow 27, 1487-1503.

Goncalves, E. and Zeidan, D. (2018), 'Simulation of compressible two-phase flows using a void ratio transport equation', Communication in Computational Physics 24(1), 167203. 
Goncalves, E., Hoarau, Y. and Zeidan, D. (2018), 'Simulation of shock-induced bubble collapse using a four-equation model', Shock Waves pp. 1-14.

Haas, J. and Sturtevant, B. (1987), 'Interaction of weak shock waves with cylindrical and spherical gas inhomogeneities', Journal of Fluid Mechanics 181, 41-76.

Hawker, N. and Ventikos, Y. (2012), 'Interaction of a strong shockwave with a gas bubble in a liquid mediam: a numerical study', Journal of Fluid Mechanics 701, 59-97.

Hu, X., Khoo, B., Adams, N. and Huang, F. (2006), 'A conservative interface method for compressible flow', Journal of Computational Physics 219, 553-578.

Jamaluddin, A., Ball, G., Turangan, C. and Leighton, T. (2011), 'The collapse of single bubbles and approximation of the far-field acoustic emissions for cavitation induced by shock wave lithotripsy', Journal of Fluid Mechanics 677, 305-341.

Janan, M. T. and Marjani, A. E. (2007), 'A flow solver for the Euler and Navier-Stokes equations for multi-phase flows with a stiffened gas equation of state', International Journal of Numerical Method for Heat and Fluid Flow 17(8), 823-835.

Johnsen, E. and Colonius, T. (2009), 'Numerical simulations of non-spherical bubble collapse', Journal of Fluid Mechanics 629, 231-262.

Kapila, A., Menikoff, R., Bdzil, J., Son, S. and Stewart, D. (2001), 'Two-phase modeling of deflagration-to-detonation transition in granular materials: reduced equations', Physics of fluids 13(10), 3002-3024.

Kunz, R., Boger, D., Stinebring, D., Chyczewski, T., Lindau, J., Gibeling, H., Venkateswaran, S. and Govindan, T. (2000), 'A preconditioned Navier-Stokes method for two-phase flows with application to cavitation prediction', Computers \& Fluids 29(8), 849-875.

Lauer, E., Hu, X., Hickel, S. and Adams, N. (2012), 'Numerical investigation of collapsing cavity arrays', Physics of Fluids 24, 052104. 
Layes, G., Jourdan, G. and Houas, L. (2009), 'Experimental study on a plane shock wave accelerating a gas bubble', Physics of Fluids 21, 074102.

Lei, X. and Li, J. (2018), 'A non-oscillatory energy-splitting method for the computation of compressible multi-fluid flows', Physics of fluids 30, 040906.

Mahdi, M., Shams, M. and Ebrahimi, R. (2010), 'Effects of heat transfer on the strength of shock waves emitted upon spherical bubble collapse', International Journal of $\mathrm{Nu}$ merical Method for Heat and Fluid Flow 20(4), 372-391.

Merouani, S., Hamdaoui, O., Rezgui, Y. and Guemini, M. (2014), 'Theoretical estimation of the temperature and pressure within collapsing acoustical bubbles', Ultrasonics Sonochemistry 21, 53-59.

Metayer, O. L., Massoni, J. and Saurel, R. (2004), 'Elaborating equations of state of a liquid and its vapor for two-phase flow models', Int. Journal of Thermal Sciences 43, 265-276.

Murrone, A. and Guillard, H. (2005), 'A five equation reduced model for compressible two phase flows problems', Journal of Computational Physics 202(2), 664-698.

Nourgaliev, R., Dinh, T. and Theofanous, T. (2006), 'Adaptive characteristics-based matching for compressible multifluid dynamics', Journal of Computational Physics 213, 500-529.

Ozlem, M., Schwendeman, D., Kapila, A. and Henshaw, W. (2012), 'A numerical study of shock-induced cavity collapse', Shock Waves 22, 89-117.

Plesset, M. and Chapman, R. (1971), 'Collapse of an initially spherical vapour cavity in the neighbourhood of a solid boundary', Journal of Fluids Mechanics 47, 283-290.

Saurel, R., Boivin, P. and Metayer, O. L. (2016), 'A general formulation for cavitating, boiling and evaporating flows', Computers \& Fluids 128, 53-64. 
Saurel, R., Petitpas, F. and Abgrall, R. (2008), 'Modelling phase transition in metastable liquids: application to cavitating and flashing flows', Journal of Fluid Mechanics 607, 313-350.

Shyue, K. (1998), 'An efficient shock-capturing algorithm for compressible multicomponent problems', Journal of Computational Physics 142, 208-242.

Shyue, K. (2006), 'A volume-fraction based algorithm for hybrid barotropic and nonbarotropic two-fluid flow problems', Shock Waves 15, 407-423.

Supponen, O., Obreschkow, D., Kobel, P., Tinguely, M., Dorsaz, N. and Farhat, M. (2017), ‘Shock waves from nonspherical cavitation bubbles', Physical Review Fluids 2, 093601.

Terashima, H. and Tryggvason, G. (2009), 'A front-tracking/ghost-fluid method for fluid interfaces in compressible flows', Journal of Computational Physics 228(11), 4012-4037.

Terashima, H., Kawai, S. and Koshi, M. (2013), 'Consistent numerical diffusion terms for simulating compressible multicomponent flows', Computers \& Fluids 88, 484-495.

Toro, E., Spruce, M. and Speares, W. (1994), 'Restoration of the contact surface in the HLL-Riemann solver', Shock Waves 4, 25-34.

Turangan, C., Jamaluddin, A., Ball, G. and Leighton, T. (2008), 'Free-lagrange simulations of the expansion and jetting collapse of air bubbles in water', Journal of Fluid Mechanics 598, 1-25.

Utkin, P. (2019), 'Numerical simulation of shock wave - dense particles cloud interaction using Godunov solver for Baer-Nunziato equations', International Journal of Numerical Method for Heat and Fluid Flow.

Wallis, G. (1967), 'One-dimensional two-phase flow', New York: McGraw-Hill.

Xie, W., Liu, T. and Khoo, B. (2006), 'Application of a one-fluid model for large scale homogeneous unsteady cavitation: the modified Schmidt mode', Computers \& Fluids 35, 1177-1192. 

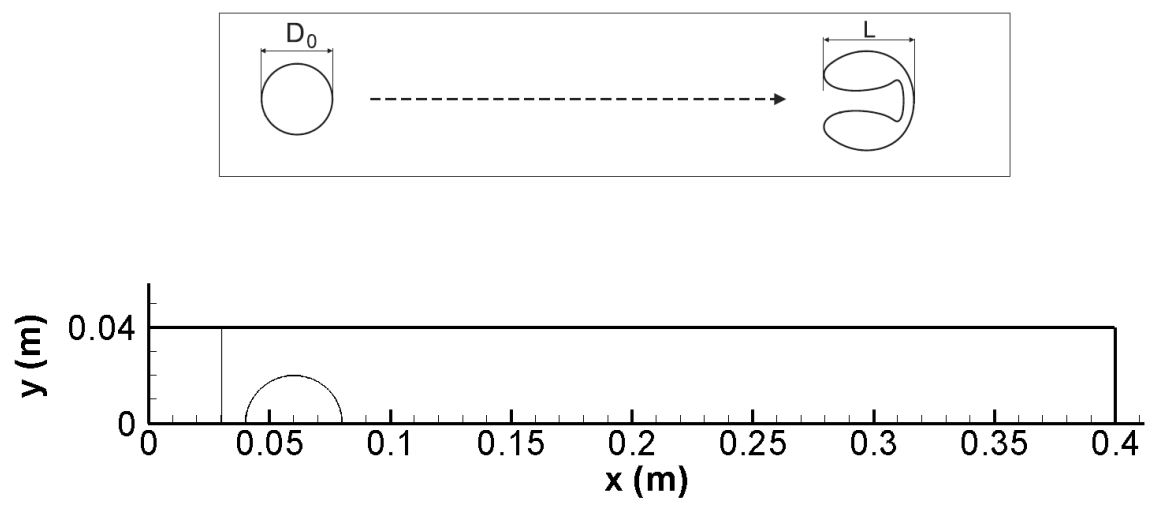

Figure 1: Definition of the bubble elongation $L$ (top) and initial situation for the helium bubble impact (down). 


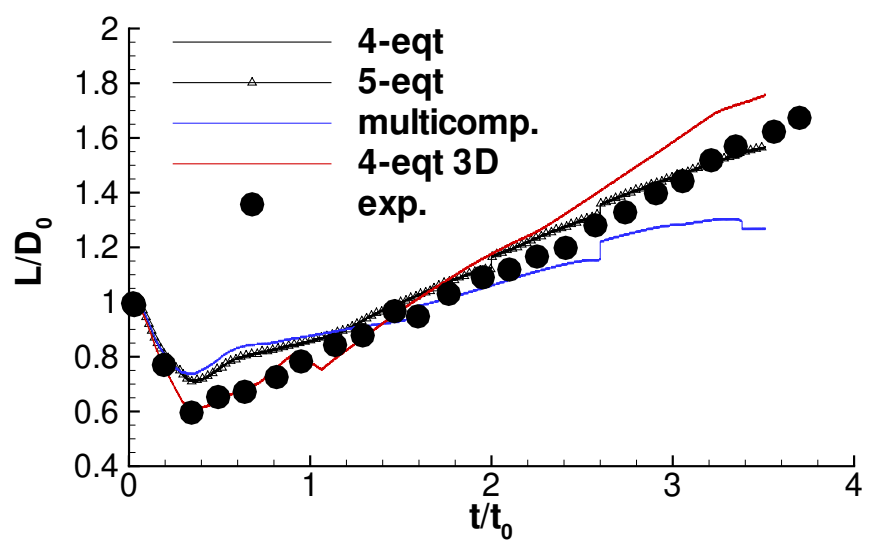

Figure 2: Evolution of the bubble elongation $L / D_{0}$ function of the dimensionless time $t / t_{0}$. Comparison between the different models and experimental data. 

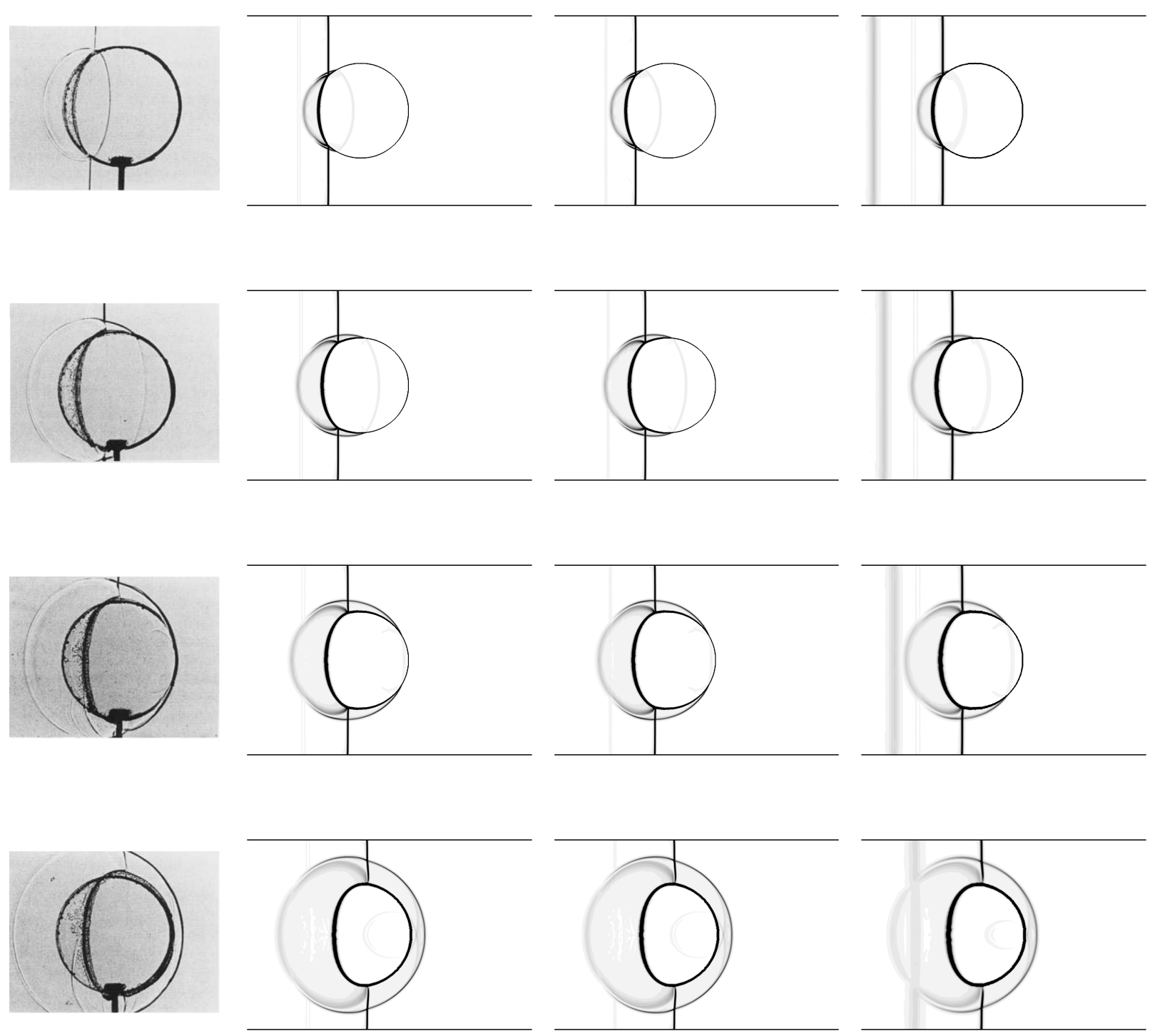

Figure 3: Evolution of the density gradient modulus at different dimensionless times: $t / t_{0}=0.09,0.12,0.14$ and 0.19 from top to down. 2D simulations performed with the 4-equation model (2nd column), the 5-equation model (3rd column) and the multicomponent model (4th column). Comparison with experimental visualizations from Haas and Sturtevant (1987) (1st column). 

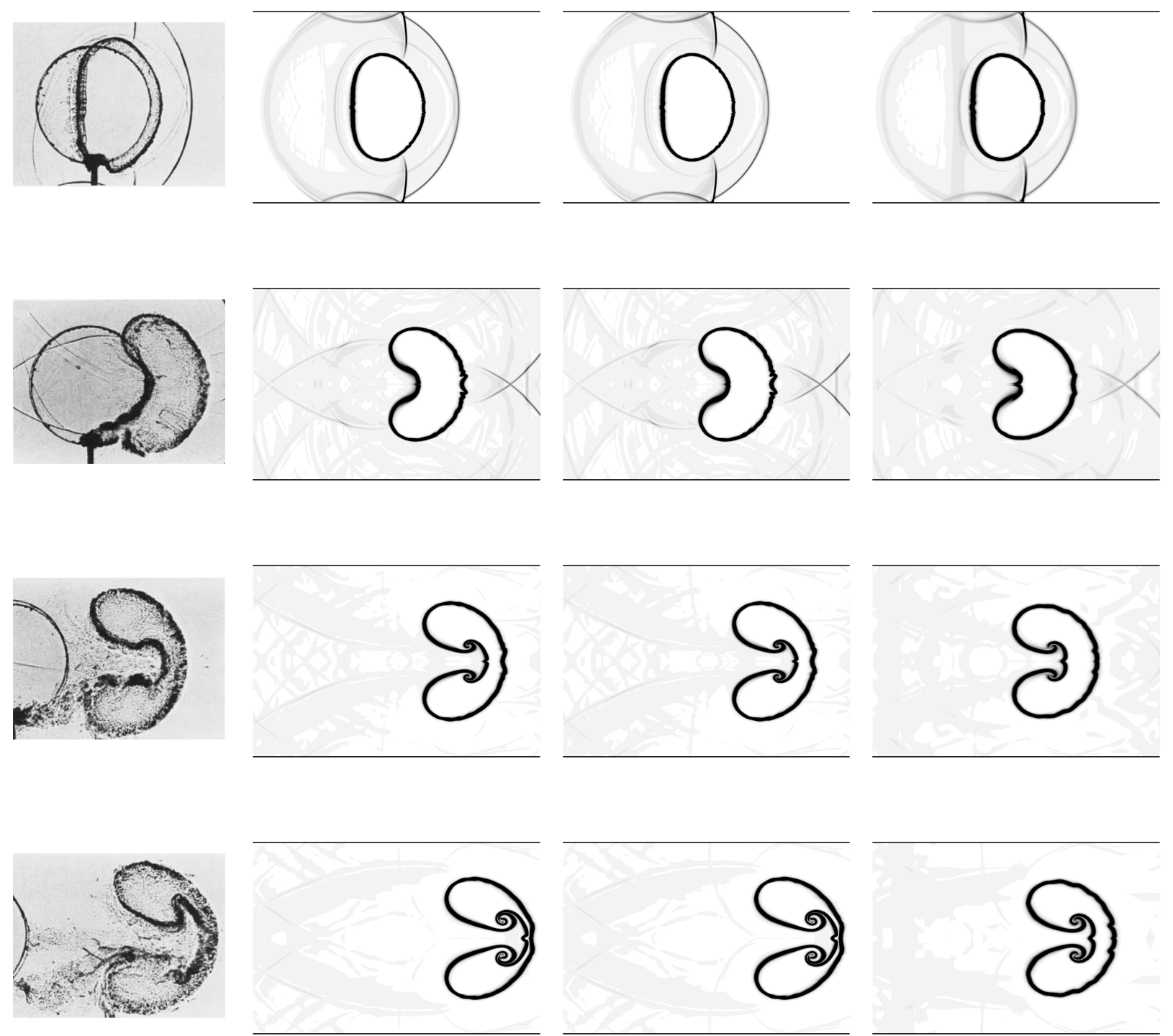

Figure 4: Evolution of the density gradient modulus at different dimensionless times: $t / t_{0}=0.26,0.59,0.94$ and 1.17 from top to down. 2D simulations performed with the 4-equation model (2nd column), the 5-equation model (3rd column) and the multicomponent model (4th column). Comparison with experimental visualizations from Haas and Sturtevant (1987) (1st column). 
a)

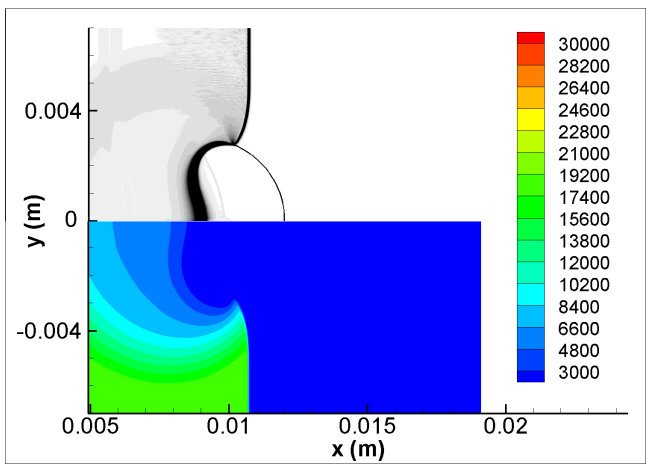

b)

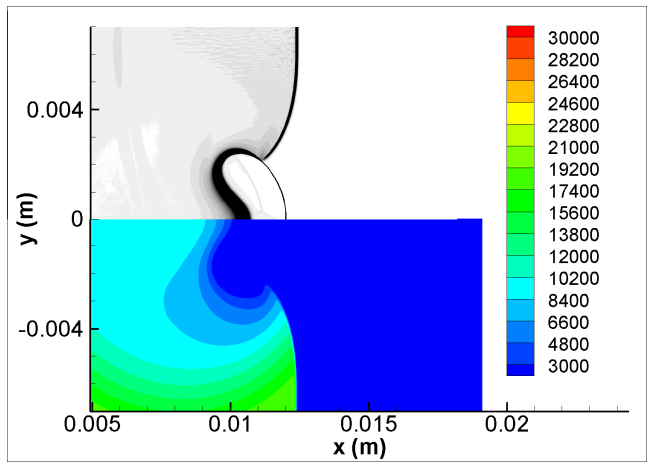

c)

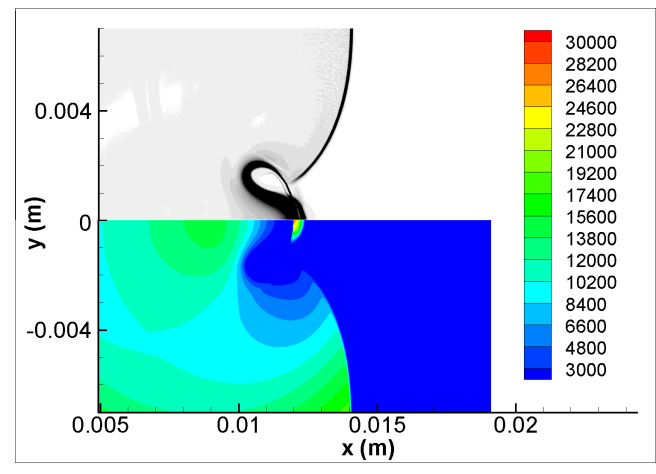

d)

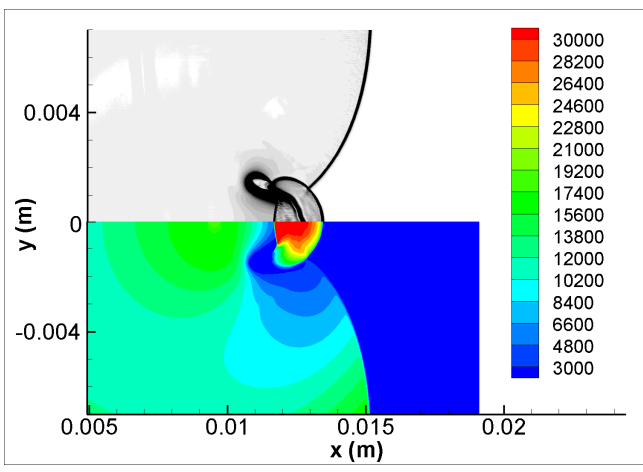

e)

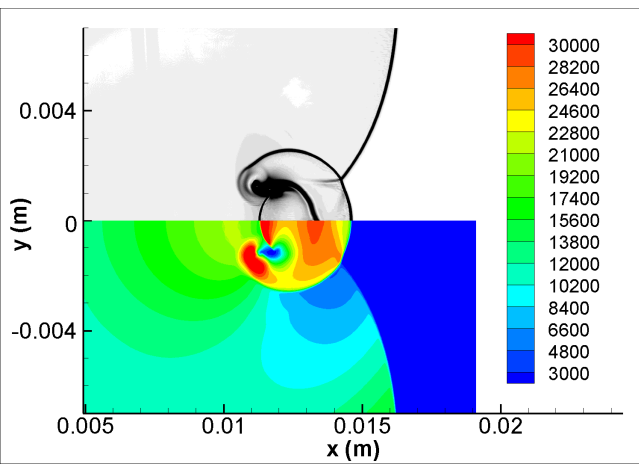

f)

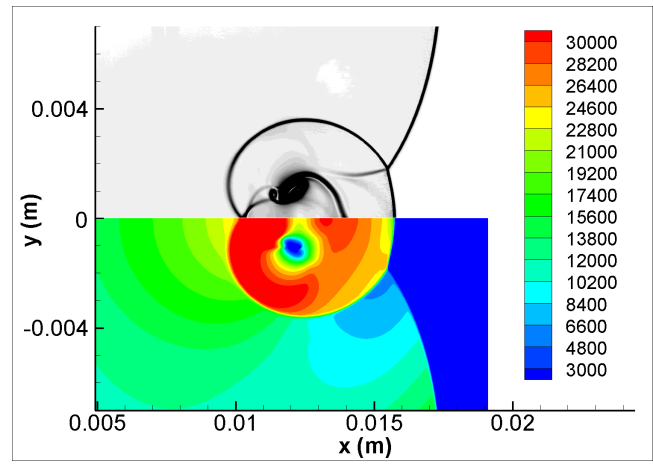

Figure 5: Evolution of the density gradient modulus and pressure field (in bar) at times: a) $t=2.4 \mu \mathrm{s}$, b) $t=3 \mu \mathrm{s}$, c) $t=3.6 \mu \mathrm{s}, \mathrm{d}) t=4 \mu \mathrm{s}$, e) $t=4.4 \mu \mathrm{s}$, and f) $t=4.8 \mu \mathrm{s}$. 2D simulations performed with the 5 -equation model. 


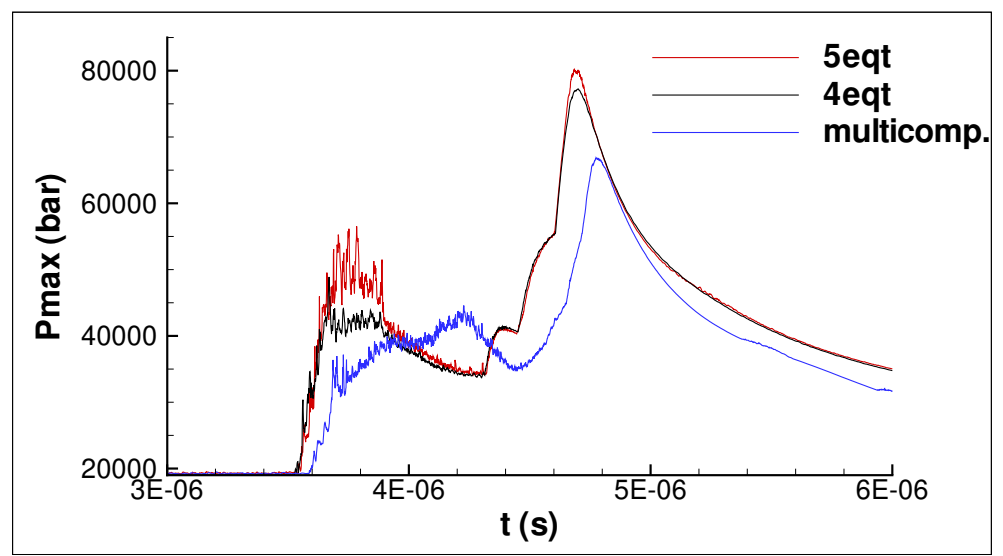

Figure 6: Evolution of the maximum pressure (in bar) reached during the collapse obtained with the three models. $2 \mathrm{D}$ results. 

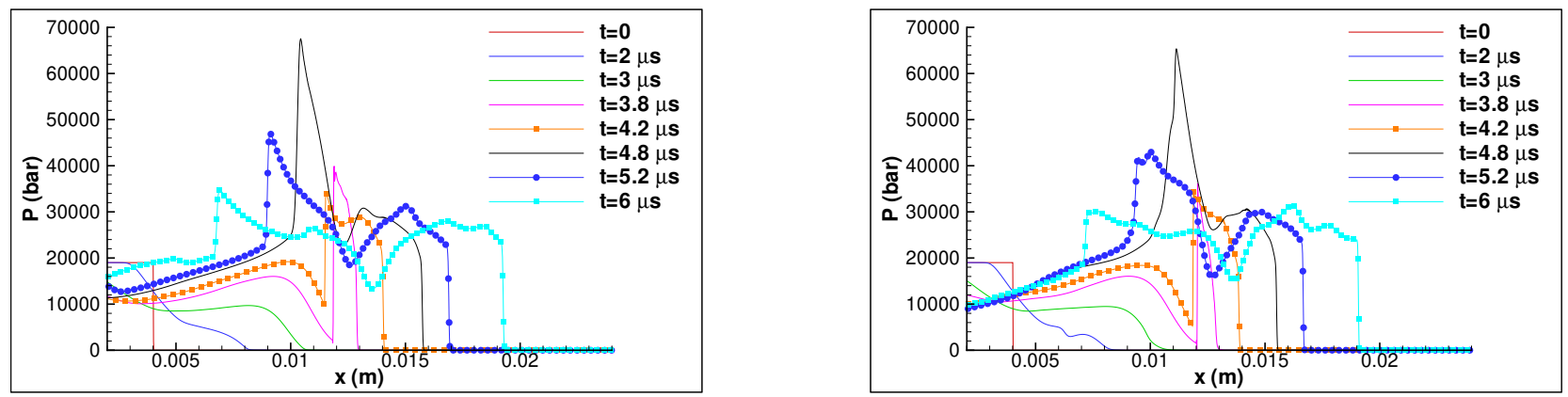

Figure 7: Evolution of the pressure during the bubble collapse on the symmetry axis for the 4-equation model (left) and the multicomponent model (right). 2D results. 

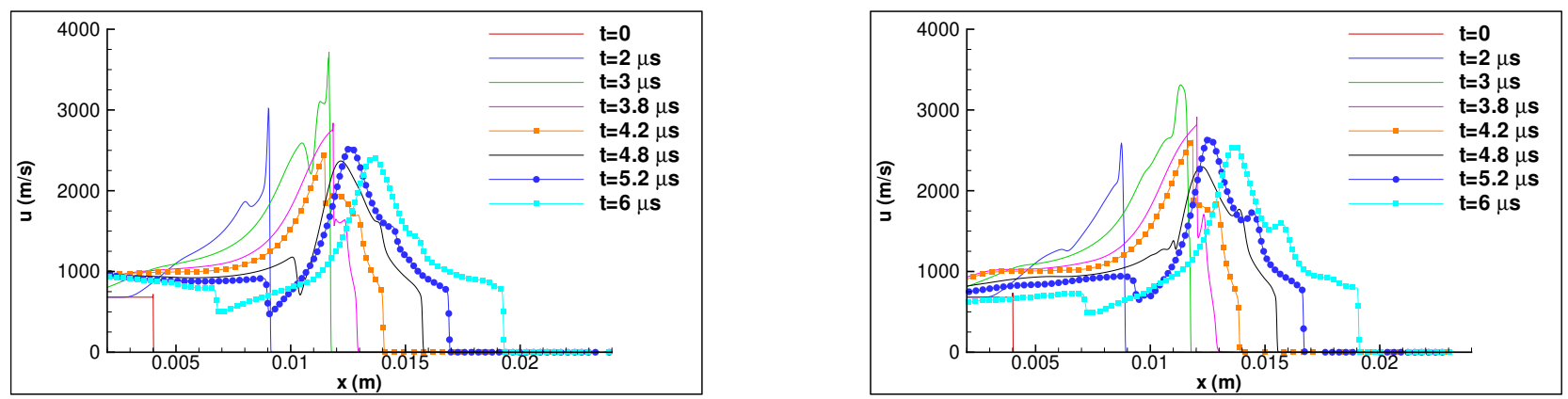

Figure 8: Evolution of the axial velocity during the bubble collapse on the symmetry axis for the 4-equation model (left) and the multicomponent model (right). 2D results. 

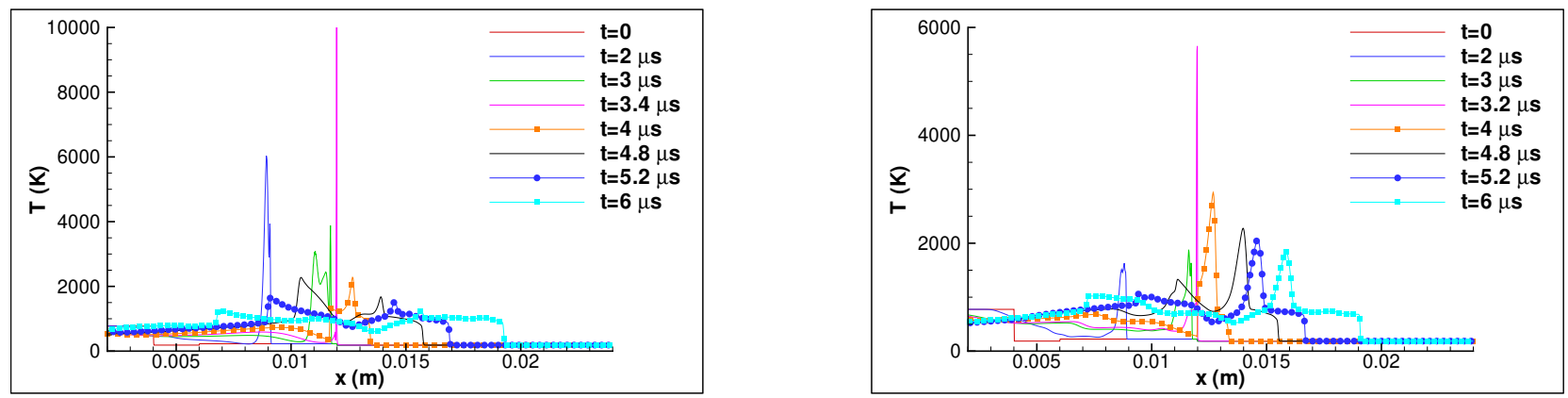

Figure 9: Evolution of the mixture temperature during the bubble collapse on the symmetry axis for the 4-equation model (left) and the multicomponent model (right). 2D results. 


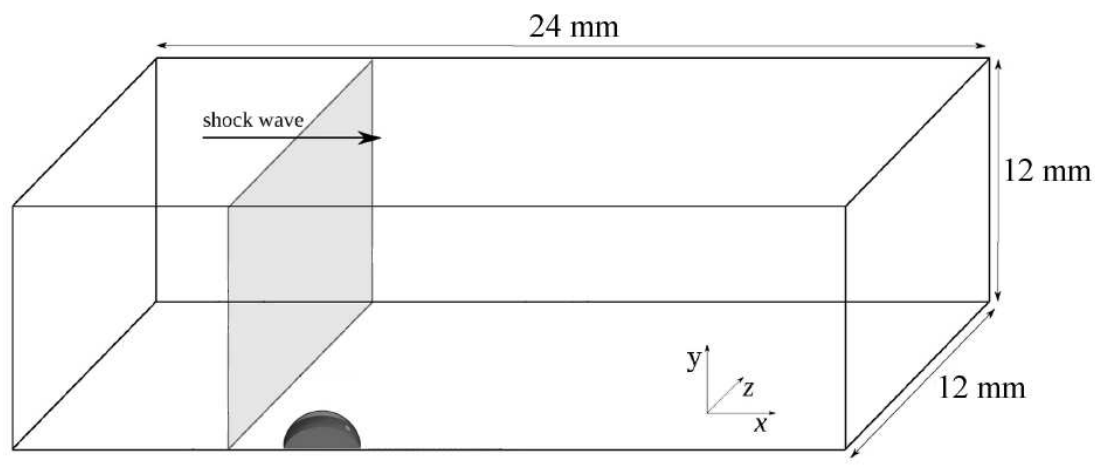

Figure 10: Initial situation for the three-dimensional simulation. 


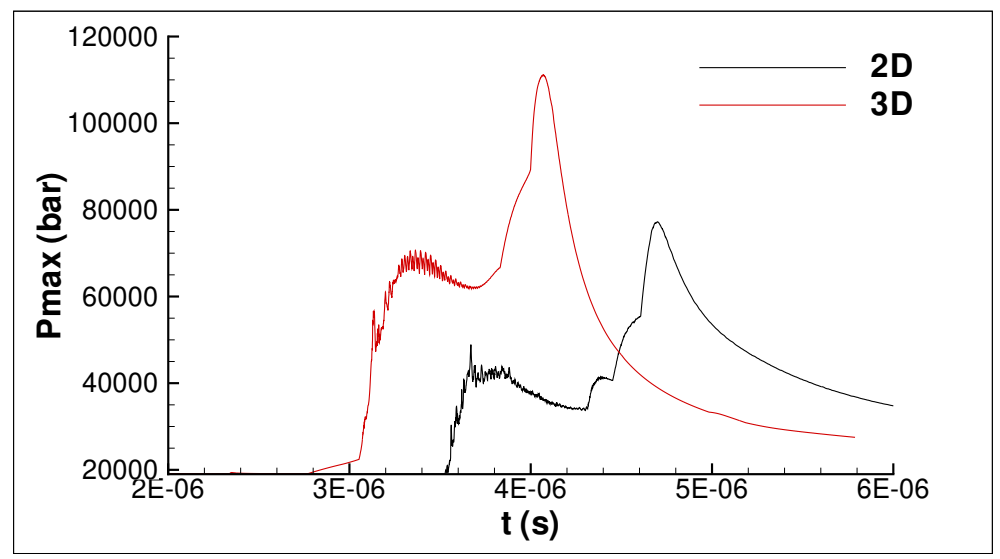

Figure 11: Evolution of the maximum pressure reached during the collapse. Comparison between 2D and 3D simulations obtained with the 4-equation model. 
a)

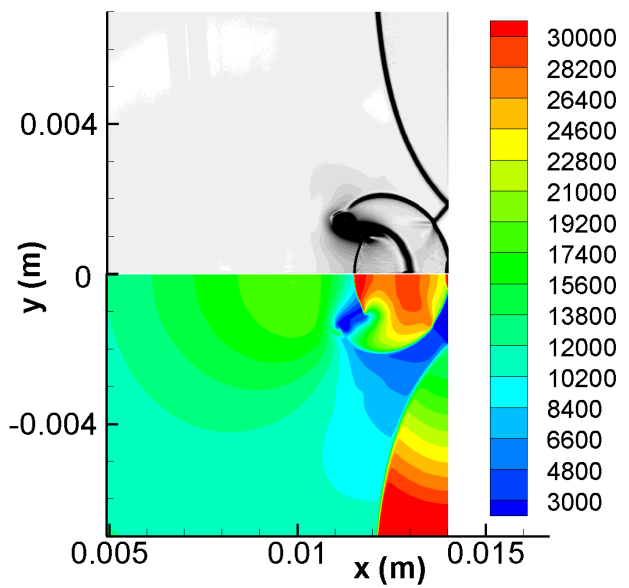

b)

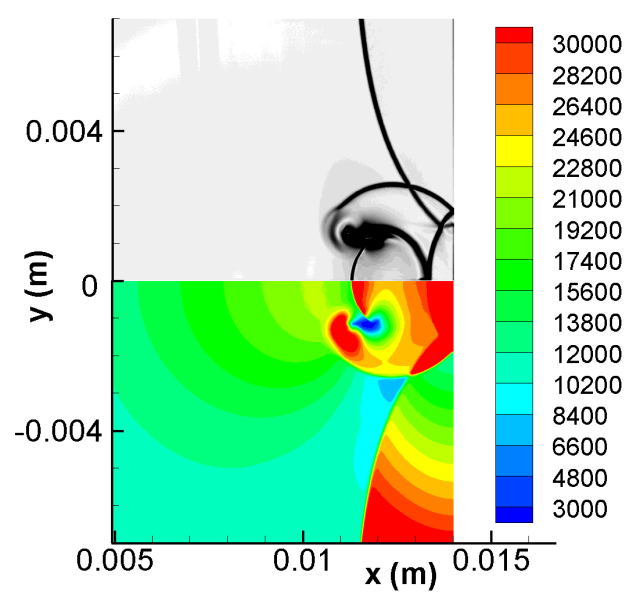

c)

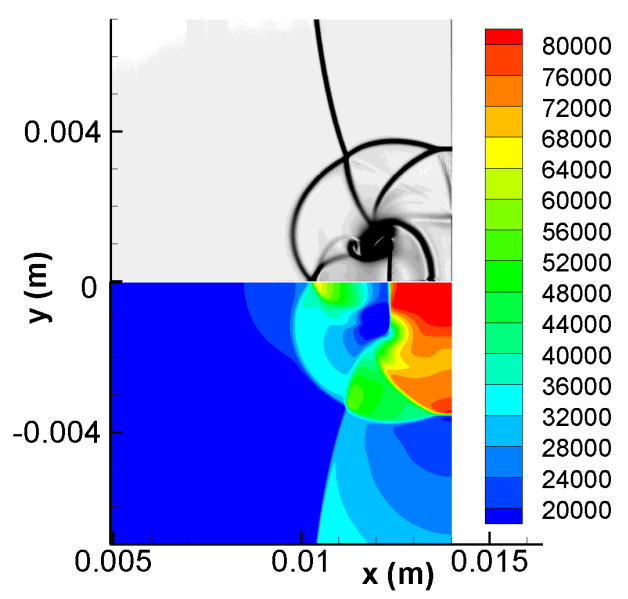

d)

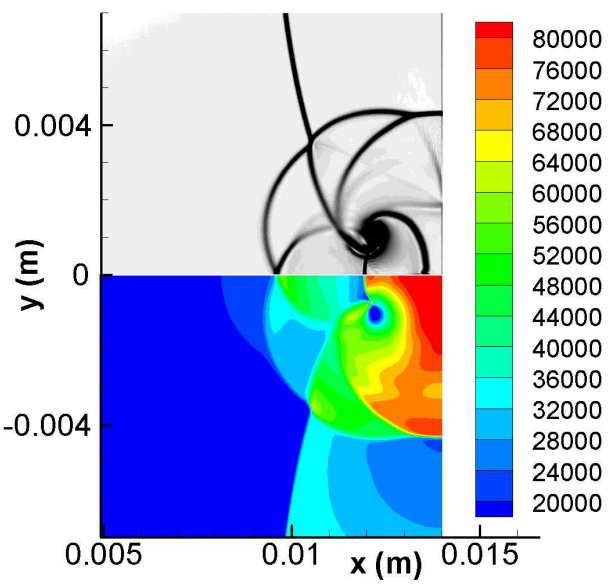

e)

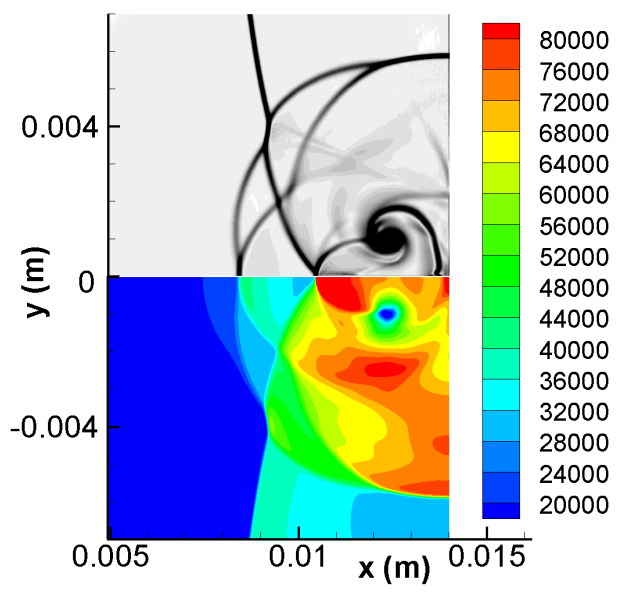

f)

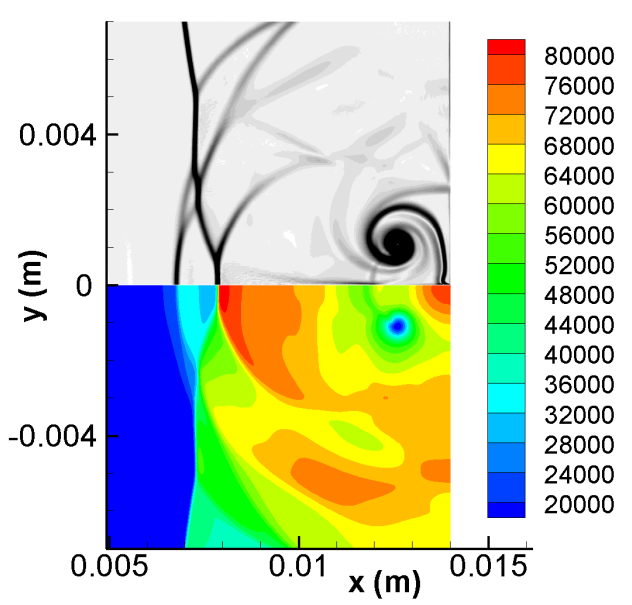

Figure 12: Evolution of the density gradient modulus and pressure field (in bar) at times:

a) $t=4.2 \mu \mathrm{s}, \mathrm{b}) t=4.4 \mu \mathrm{s}, \mathrm{c}) t=4.8 \mu \mathrm{s}, \mathrm{d}) t=5 \mu \mathrm{s}, \mathrm{e}) t=5.4 \mu \mathrm{s}$, and f) $t=6 \mu \mathrm{s} .2 \mathrm{D}$ simulation performed with the 4-equation model. Collapse near a wall with $L / R=1.66$. 

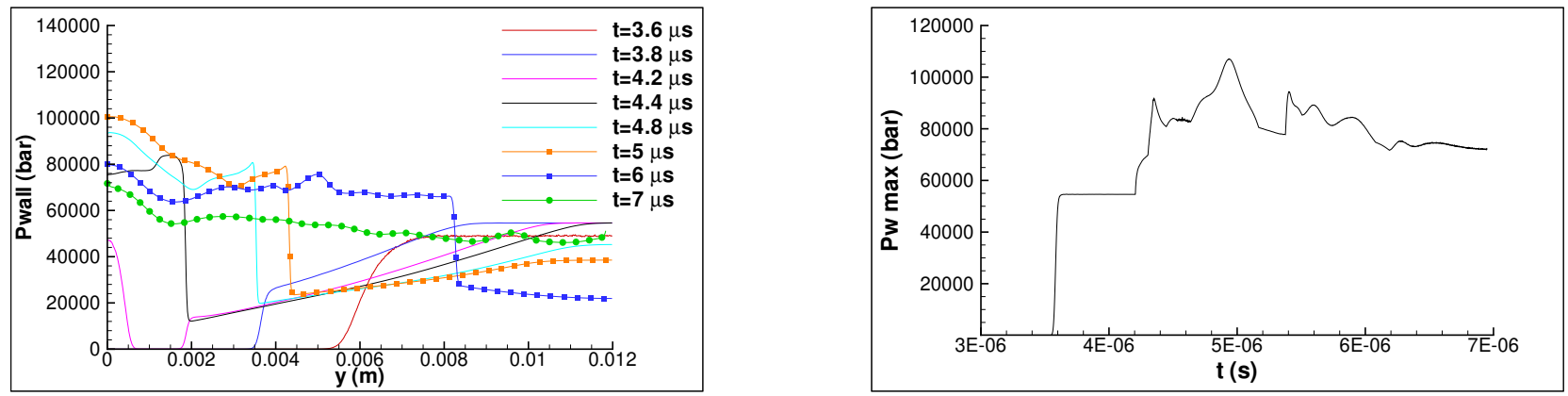

Figure 13: Evolution of the wall pressure on the symmetry axis (left) and the maximum wall pressure (right). 2D simulation performed with the 4-equation model. Collapse near a wall with $L / R=1.66$. 
a)

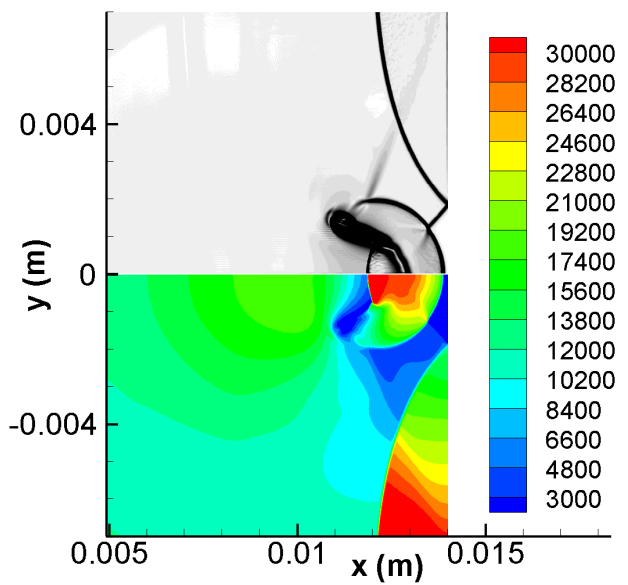

b)

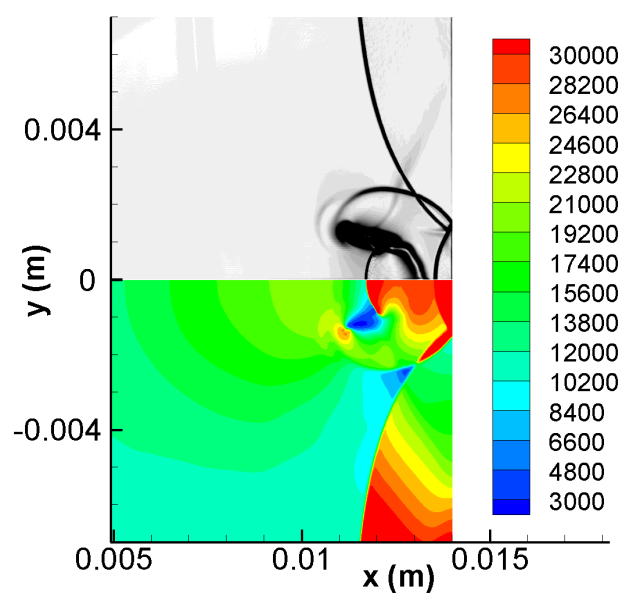

c)

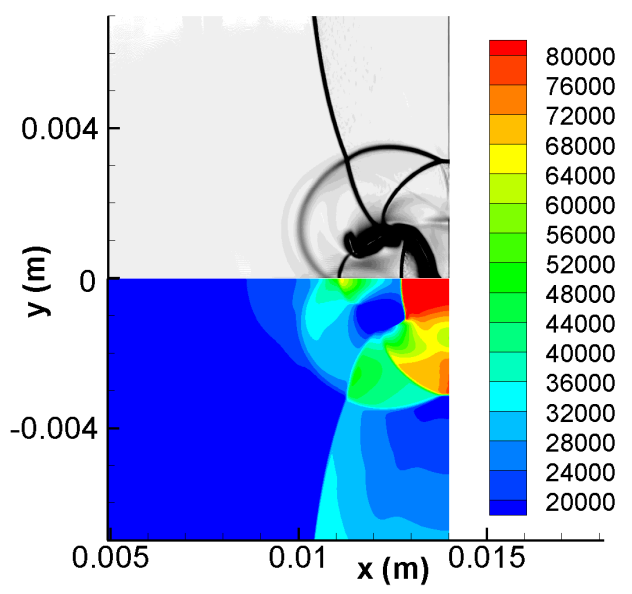

d)

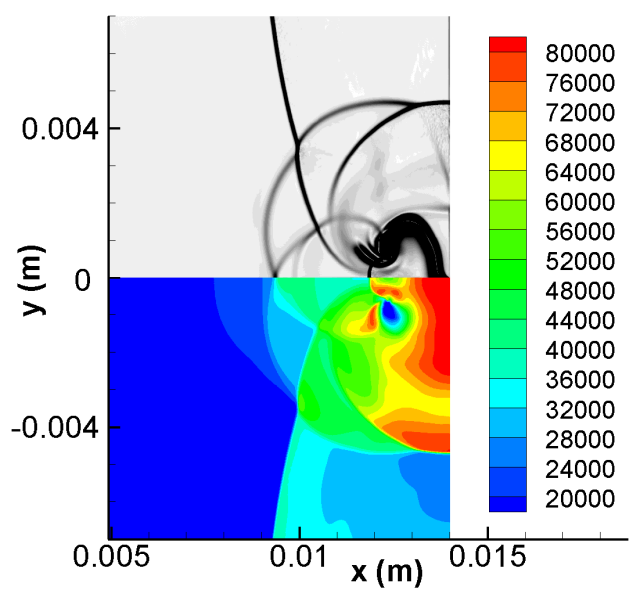

e)

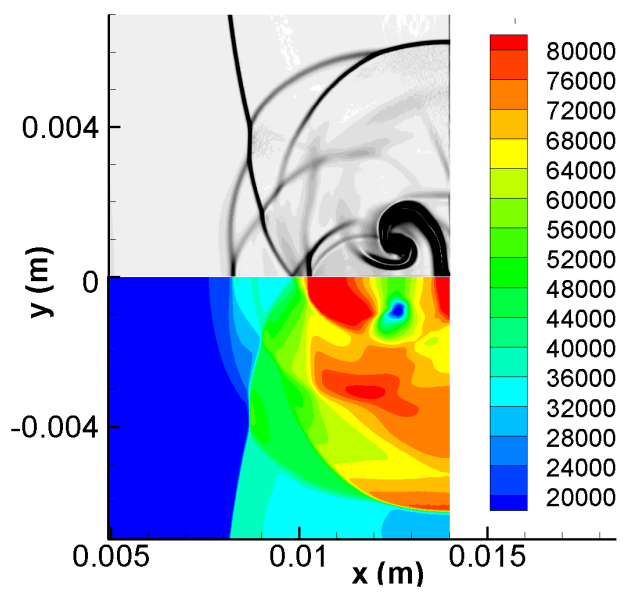

f)

Figure 14: Evolution of the density gradient modulus and pressure field (in bar) at times:

a) $t=4.2 \mu \mathrm{s}, \mathrm{b}) t=4.4 \mu \mathrm{s}, \mathrm{c}) t=4.8 \mu \mathrm{s}, \mathrm{d}) t=5.2 \mu \mathrm{s}, \mathrm{e}) t=5.6 \mu \mathrm{s}$, and f) $t=6.0$ $\mu \mathrm{s} .2 \mathrm{D}$ simulation performed with the multicomponent model. Collapse near a wall with $L / R=1.66$. 

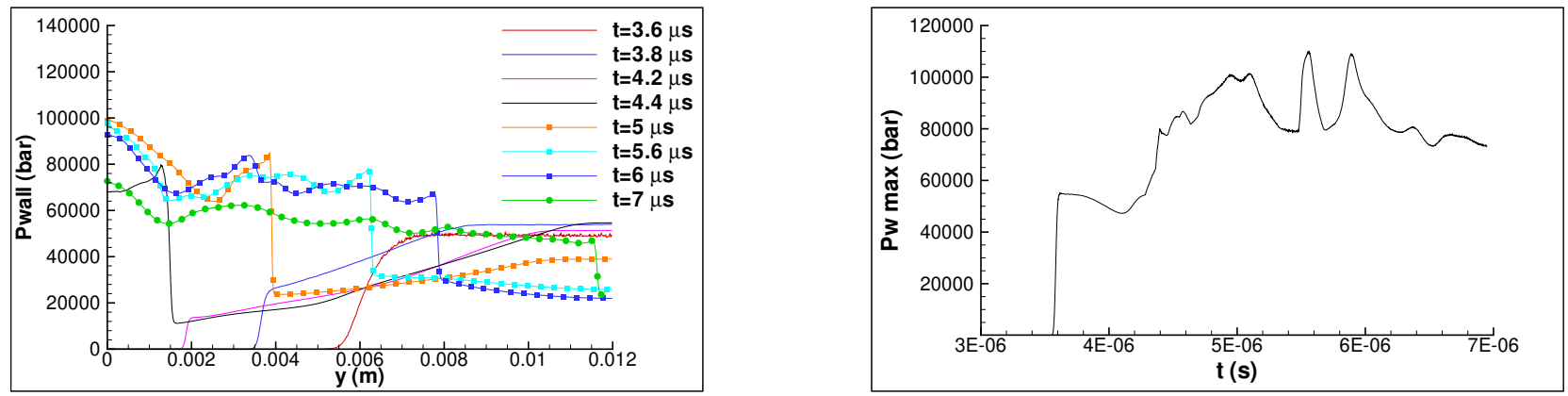

Figure 15: Evolution of the wall pressure on the symmetry axis (left) and the maximum wall pressure (right). 2D simulation performed with the multicomponent model. Collapse near a wall with $L / R=1.66$. 

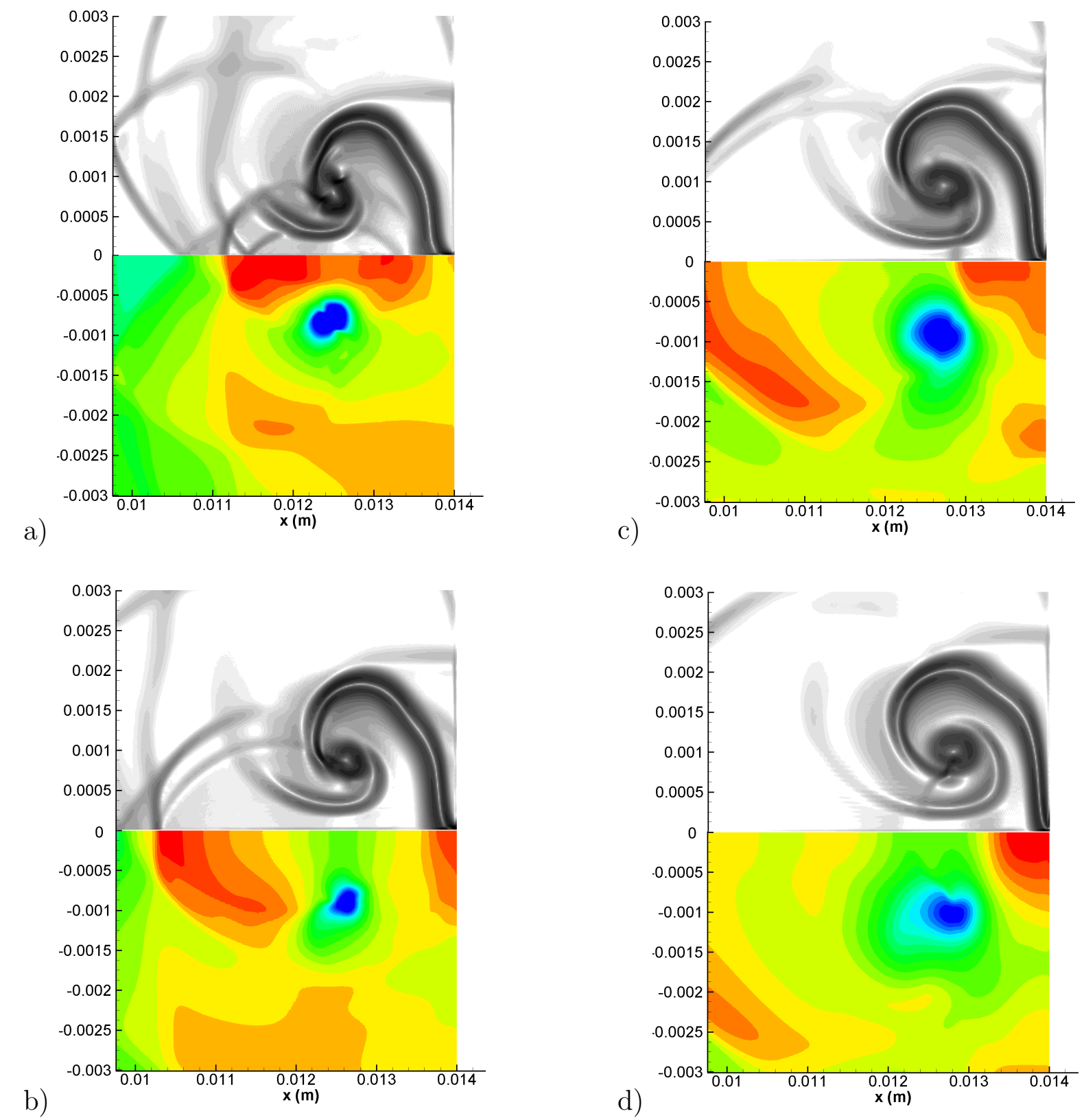

Figure 16: Enlargement of the density gradient modulus and pressure field (in bar) at times: a) $t=5.4 \mu \mathrm{s}$, b) $t=5.6 \mu \mathrm{s}$, c) $t=5.8 \mu \mathrm{s}$, and d) $t=6.0 \mu \mathrm{s} .2 \mathrm{D}$ simulation performed with the multicomponent model. Collapse near a wall with $L / R=1.66$. 
a)

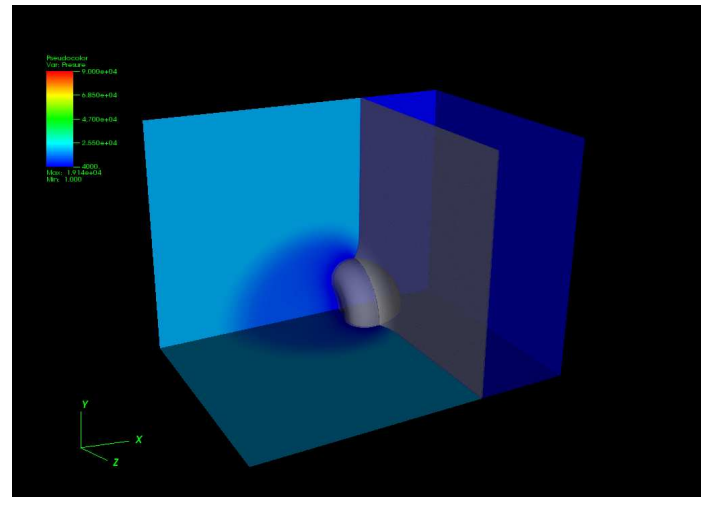

b)

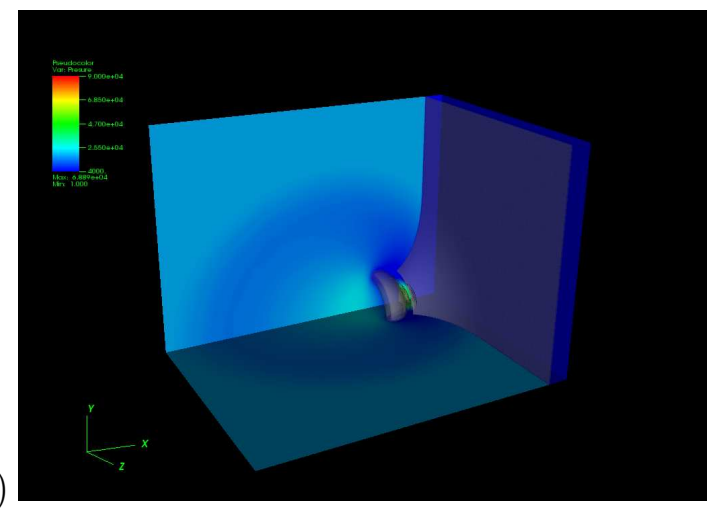

c)

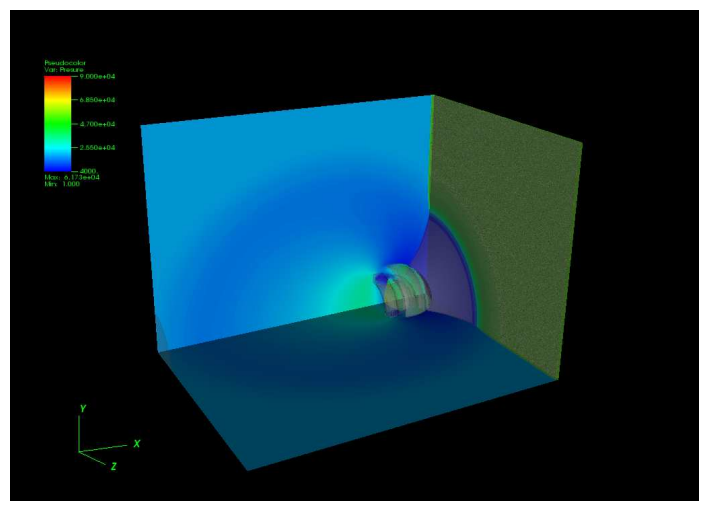

d)

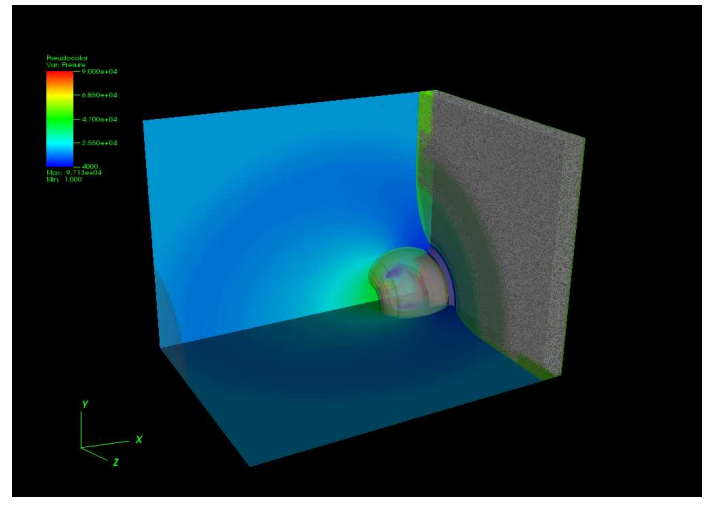

e)

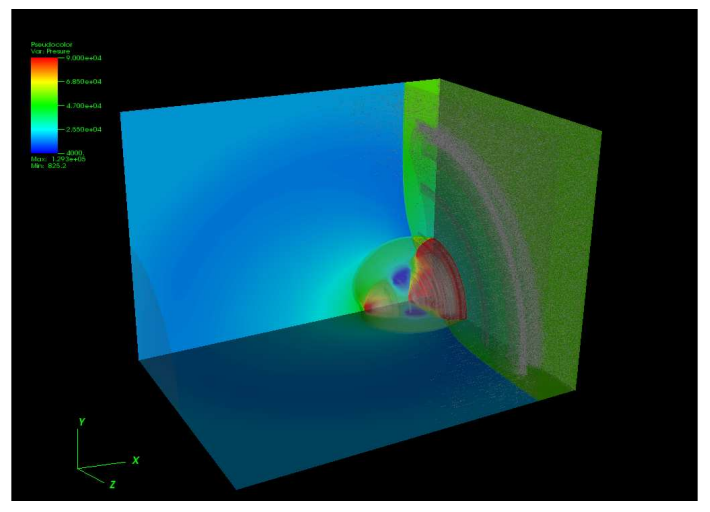

f)

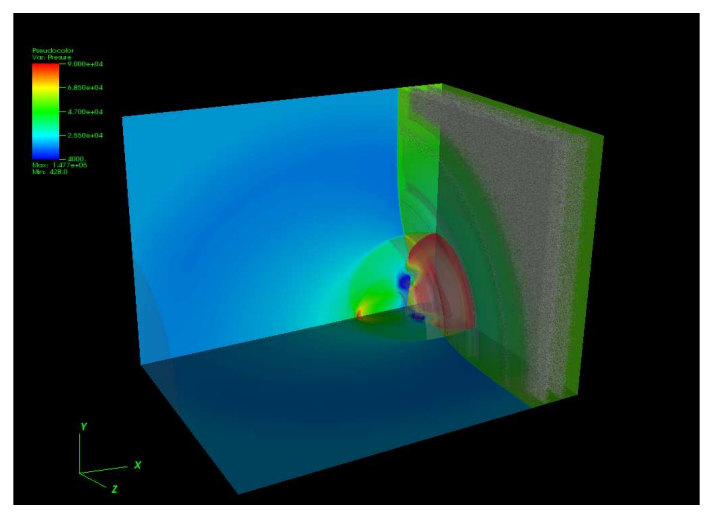

Figure 17: Evolution of the pressure field (in bar) and isosurfaces of the density at times:

a) $t=2.15 \mu \mathrm{s}$, b) $t=3.25 \mu \mathrm{s}$, c) $t=3.65 \mu \mathrm{s}$, d) $t=3.9 \mu \mathrm{s}$, e) $t=4.15 \mu \mathrm{s}$, and f) $t=4.25 \mu \mathrm{s}$. 3D simulation performed with the 4-equation model. Collapse near a wall with $L / R=1.66$. 
a)

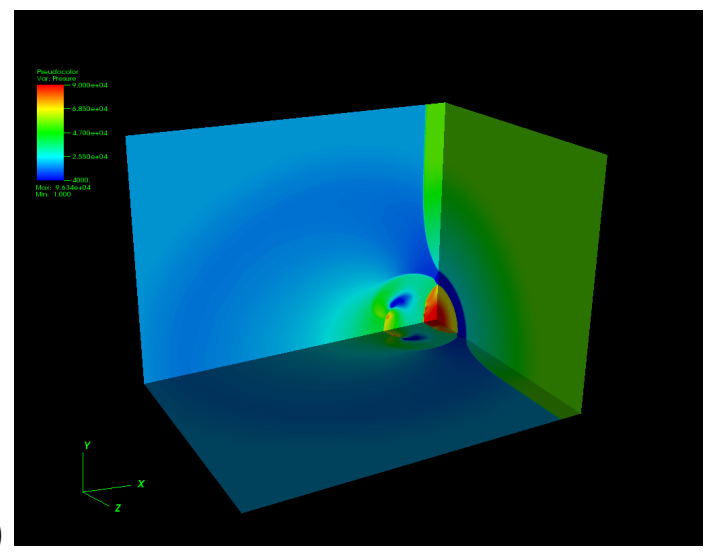

b)

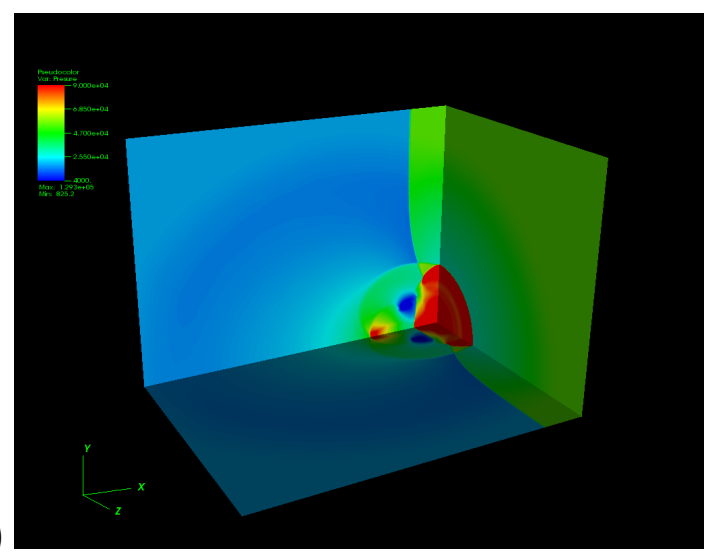

c)

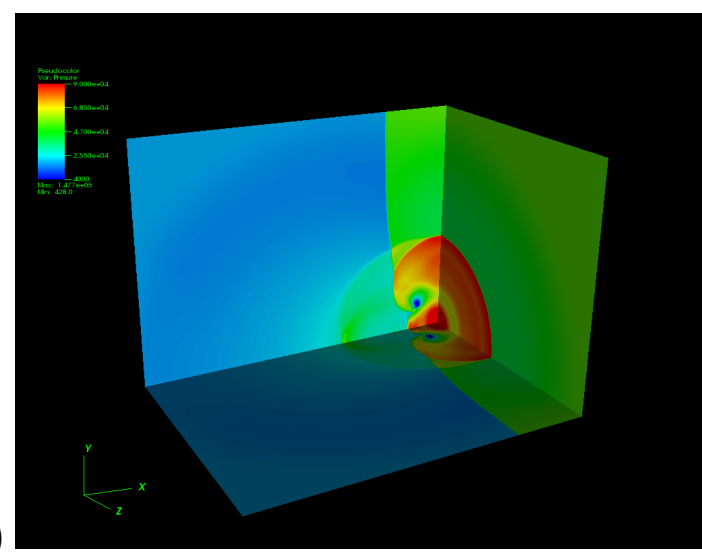

d)

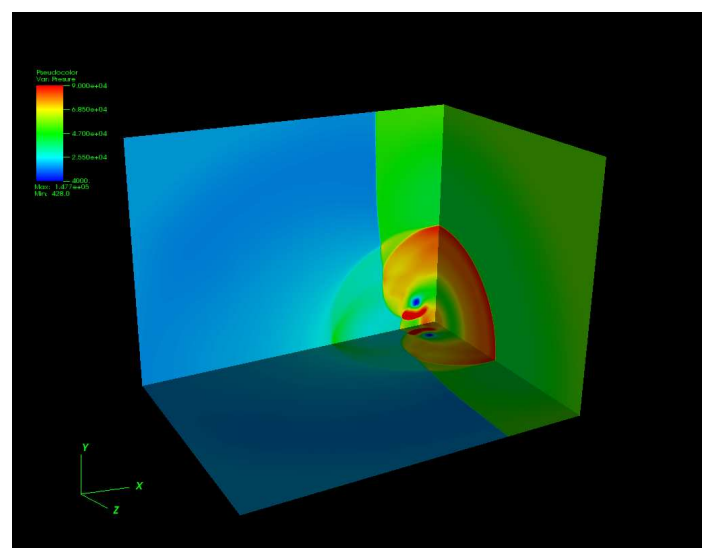

e)

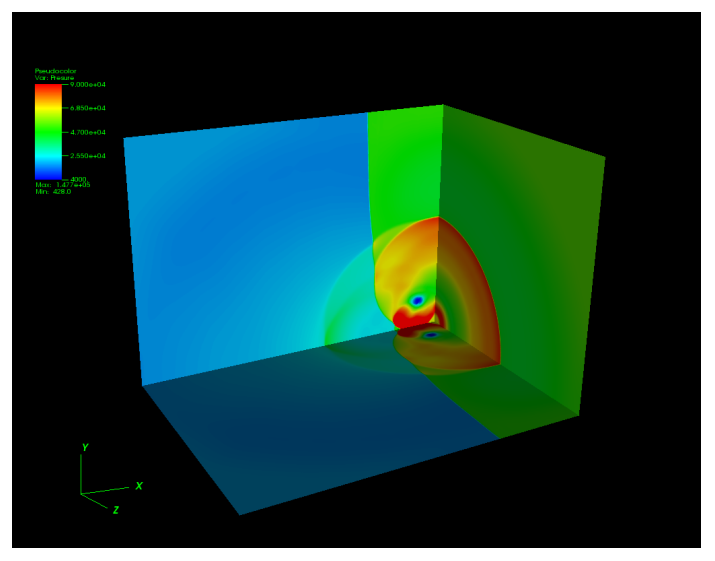

f)

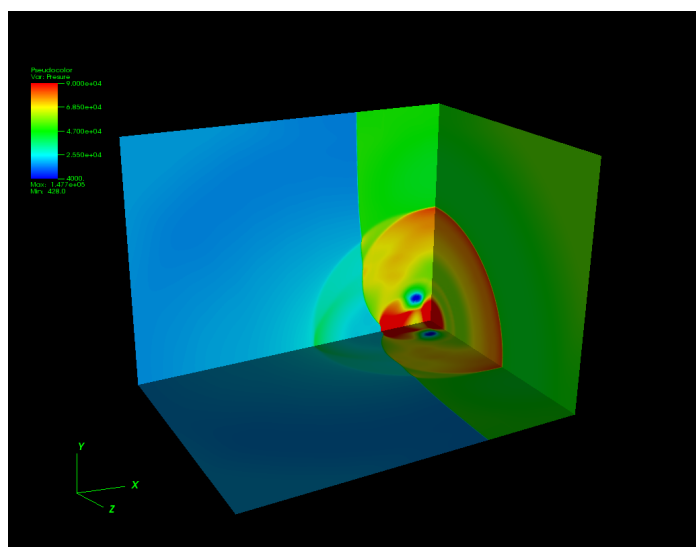

Figure 18: Evolution of the pressure field (in bar) at times: a) $t=3.9 \mu \mathrm{s}, \mathrm{b}) t=4.15 \mu \mathrm{s}$, c) $t=4.5 \mu \mathrm{s}, \mathrm{d}) t=4.6 \mu \mathrm{s}$, e) $t=4.75 \mu \mathrm{s}$, and f) $t=4.85 \mu \mathrm{s}$. 3D simulation performed with the 4-equation model. Collapse near a wall with $L / R=1.66$. 


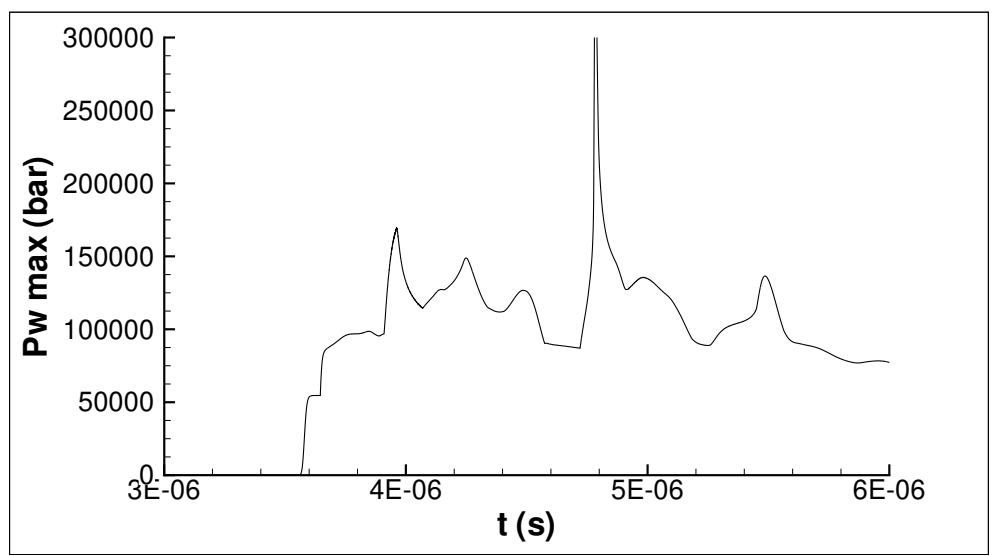

Figure 19: Evolution of the maximum wall pressure reached during the collapse. 3D simulation performed with the 4 -equation model. Collapse near a wall with $L / R=1.66$. 


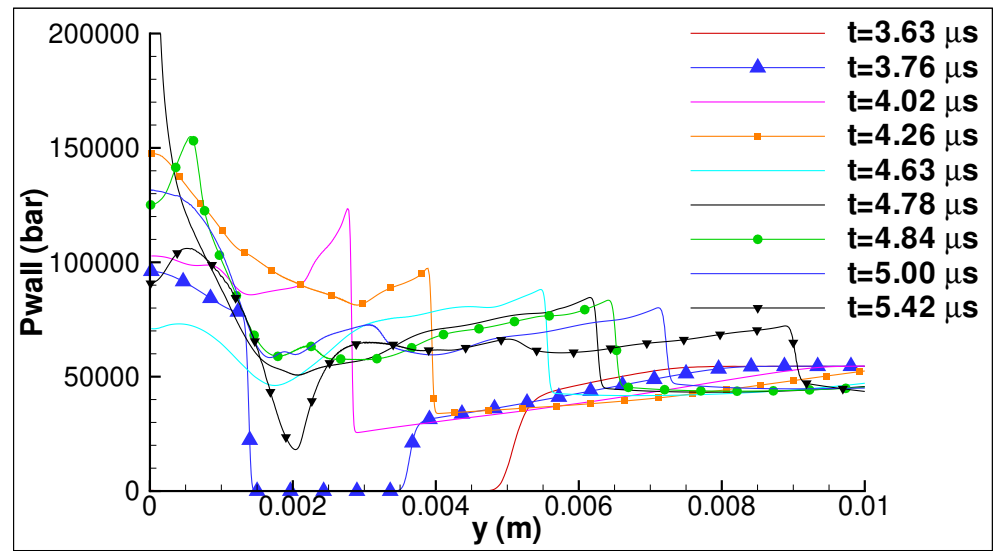

Figure 20: Evolution of the wall pressure on the symmetry axis at different times. 3D simulation performed with the 4 -equation model. Collapse near a wall with $L / R=1.66$. 